\title{
ANALISIS DE UNA EXPERIENCIA: La Misión Pedagógica Alemana
}

"El tiempo que perdiste por tu rosa hace que tu rosa sea tan importante". El Principito.

\section{María Cristina Rojas de Ferro **}

Realizar una evaluación como la propuesta, la de la Misión Pedagógica Alemana, entraña un significado muy especial. Es en primer lugar, mirar retrospectivamente la labor conjunta de un grupo de colombianos que durante diez años (1968-1978) trabajó con expertos alemanes en la reforma cualitativa de la educación primaria en Colombia. Es también recoger las experiencias de algo que se inicia con un diagnóstico amplio y consciente de la problemática que caracterizaba el sistema educativo y que cristaliza en una serie de actividades encaminadas a suplir las fallas detectadas. Es analizar en cierto modo las políticas educativas, las prioridades gubernamentales y los procesos de toma de decisiones en la medida en que se reflejan e inciden en los resultados alcanzados por la Misión; y, finalmente, es evaluar la asesoría educativa de un grupo que ya en 1872, con la llegada de la primera Misión Pedagógica Alemana, inicia su labor en la enseñanza primaria y normalista colombiana.

La complejidad de esta tarea y las múltiples actividades realizadas durante el proyecto hacen que se constituyan en objeto de este estudio tanto la situación del sistema educativo, especialmente los planes y reformas anteriores y posteriores a la misión, como los aspectos organizativos y administrativos que caracterizaron el proyecto y los resultados producidos durante la asesoría alemana. Con el fin de delimitar y ordenar la exposición, el presente artículo se ha dividido de la siguiente manera: la primera parte trata de algunos aspectos teóricos sobre lo que es la investigación evaluativa, campo dentro del cual se desarrolla este estudio; en la segunda parte se especifica el alcance y la metodología empleada en la evaluación de la Misión; los siguientes apartes presentan los resultados de la aplicación de esta metodología, para terminar con un análisis de lo que se puede aprender de esta experiencia.

\section{La investigación evaluativa:}

La investigación evaluativa es definida como la determinación de los resultados alcanzados por una actividad diseñada para cumplir algún fin u objetivo buscado (Suchmann, 1967); o como el procedimiento por el cual los programas son estudiados para definir su efectividad en el logro de una meta (Greenberg, 1971).

Las finalidades de la investigación evaluativa pueden ser varias; una de ellas es la de "probar" la legitimidad y efectividad de un programa respecto al apoyo social que se le ha prestado; otra es la de servir de medio para afectar el nivel político-decisorio hacia la

\footnotetext{
El presente artículo contiene los resultados de una investigación llevada a cabo en el Centro de Investigaciones de la Universidad Pedagógica Nacional con el apoyo de la Sociedad Alemana de Cooperación Técnica G.T.Z. y el Ministerio de Educación Nacional. Formaron parte del equipo investigador Fabio Moreno y Orlando Gutiérrez.

"Licenciada en Filosofía y Letras, Universidad Javeriana, M.D. Administración Educativa, Universidad Pedagógica Nacional; Ed. M. en Planeamiento y Política Social, Universidad de Harvard. Jefe División Desarrollo Social, Departamento Nacional de Planeación.
} 
continuación o terminación de un programa; en este caso se juzga útil si mueve la política social hacia la dirección defendida por los encargados del planeamiento (Freemann, 1977); desde una perspectiva administrativa, la investigación evaluativa se justifica por la necesidad de corroborar que aquello por lo que se pagó, se ejecutó o se está ejecutando; finalmente la evaluación es vista como una fase del planeamiento sistemático y como herramienta que provee las bases para el mejoramiento de los programas (Caro, 1971).

Respecto al campo que abarca la evaluación, ésta se divide en evaluación de proceso, diseñada para saber si un programa se está implementando de acuerdo a sus lineamientos iniciales; de impacto, relacionada con la medición del cambio resultante como consecuencia del programa; y evaluación comprehensiva que incluye tanto la de proceso como la de impacto (Freemann, 1977). Dependiendo del estado del programa sujeto a evaluación, puede ser formativa cuyo fin es mejorar, replantear o rediseñar un programa cuando aún está en marcha y sumativa diseñada para evaluar el programa cuando ha concluido o está bien establecido (Scriven, 1967). De acuerdo al tiempo en que se ejecute, la evaluación puede ser "ex ante" y ex post" según se conduzca antes o después de realizado el programa (Cain y Robinson, 1972).

La investigación evaluativa es también dividida en cuatro tipos: contexto, insumos, proceso y producto (Stuflebeam, 1971). La primera, define el marco conceptual en que han de basarse la determinación de los objetivos así como las condiciones reales del entorno, diagnosticando los problemas que obstaculizan la satisfacción y el aprovechamiento de las metas; la segunda, suministra información acerca de la manera como se utilizaron los recursos para alcanzar los objetivos; la tercera, detecta o predice los defectos en el plan de procedimiento en su implementación; la evaluación de producto mide e interpreta los logros no sólo al final del ciclo sino durante su desarrollo.

Atendiendo a los resultados que la evaluación produzca (Schumann, 1967) es clasificada en: Evaluación de esfuerzo, la cual suministra información acerca de la cantidad y la calidad de las acciones ejecutadas, asumiendo que éstas son un medio para alcanzar objetivos mayores; la evaluación de efecto mide los resultados del esfuerzo más que el esfuerzo mismo; dice cuánto se hizo de un objetivo inmediato; la adecuación del efecto se refiere al grado en que el resultado se adecua a las necesidades totales de la población; la evaluación de eficiencia contesta a la pregunta de si el programa funcionó y si existe una mejor manera de alcanzar los mismos resultados; hace referencia también a la evaluación de métodos alternativos en términos de costos; finalmente, la evaluación de proceso explicita cómo y por qué un programa funcionó o no funcionó, teniendo en cuenta tanto sus atributos y las condiciones bajo las cuales éste es más o menos exitoso, como la población a la cual va dirigido.

\section{Alcance y metodología del estudio:}

La evaluación de la Misión Pedagógica Alemana se centra fundamentalmente en la medición de los resultados alcanzados durante la asesoría del Gobierno alemán y los procedimientos empleados para su logro. Más concretamente, se persigue a través de una evaluación "ex post" dar respuesta a cuatro preguntas fundamentales: Cuál fue la contribución de la Misión Pedagógica Alemana al desarrollo educativo de la escuela primaria; cuál es la factibilidad de la implementación de un proyecto de esta naturaleza en términos de costo para el país receptor, cuáles programas y el grado en que los materiales han permanecido en las escuelas y han sido utilizados por los maestros. Queda fuera del objetivo de este trabajo la evaluación del impacto del programa, o el 
establecer en qué medida se dieron cambios cualitativos en el proceso de enseñanzaaprendizaje como consecuencia de la Asesoría Alemana (Ver Diagrama 1).

Como fuentes de información para el desarrollo de estas categorías se utilizaron documentos pertenecientes al archivo de la Misión, testimonios de personas que desde distintos niveles colaboraron con ella, una encuesta a maestros, y visitas a establecimientos de 3 departamentos del país.

\section{El entorno de la Misión Pedagógica Alemana}

La década del 60 se caracterizó por grandes cambios dentro del sistema educativo, no sólo en lo referente a la estructura curricular sino en sus aspectos cuantitativos y de mejora cualitativa. Los planes y programas que regían desde 1950 fueron remplazados por el Decreto 1710 de julio de 1963. Es la intención de la nueva reforma poner fin al sistema de educación primaria que tenía como características la desigualdad entre la zona rural y urbana en cuanto a la diferenciación de planes, contenidos básicos y duración de la escolaridad obligatoria. El acceso a la educación también se veía afectado por la desigualdad urbana-rural, reflejada no sólo en las bajas tasas de escolaridad sino en los altos índices de deserción y repitencia.

DIAGRAMA 1

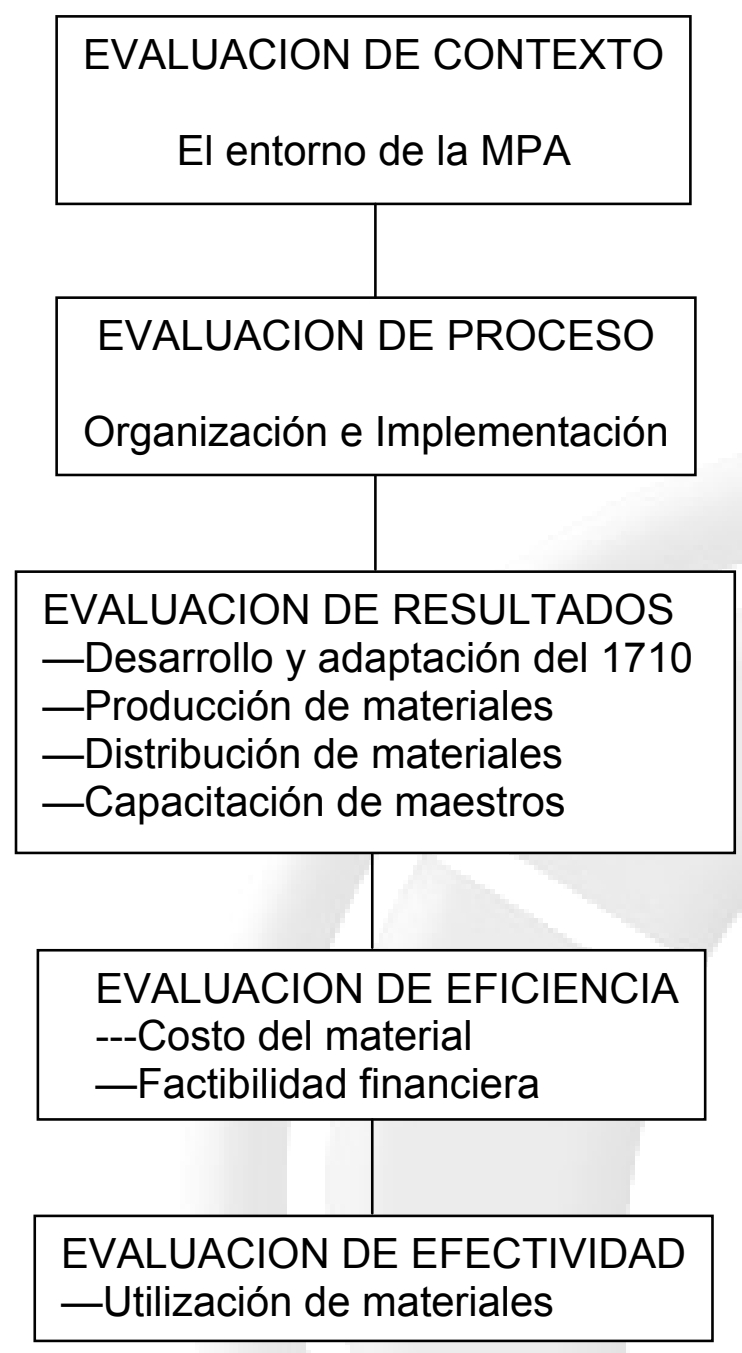


Es entonces cuando el gobierno nacional implementa el llamado "Plan de Emergencia", el cual persigue la racionalización de los recursos y la ampliación de la cobertura educativa a través de tres modalidades de educación primaria: Las escuelas de funcionamiento intensivo, en las que reduciéndose el número de horas semanales del alumno, se abrirían cursos adicionales; las escuelas de doble jornada, en las que se utilizaría una misma aula para dos grupos de alumnos con horarios especiales; y las escuelas unitarias donde con un solo maestro se dictarían los cursos de los cinco grados de primaria. Como consecuencia de estas medidas en el período de 1965 a 1974 la población de este nivel aumentó en un millón y medio de estudiantes, significando un incremento del 69o/o, comparado con un incremento de 37o/o de la población escolarizable durante el mismo tiempo.

El aspecto más preocupante era tal vez el de la calidad de la educación primaria manifestada en una baja asimilación de conocimientos por parte de los estudiantes y altos índices de deserción y repitencia. Parte de la raíz del problema se centra en la falta de preparación académica y metodológica del magisterio. Una investigación diagnóstico realizada en 1966 por quien más tarde sería el director de la Misión Pedagógica Alemana confirma esta hipótesis: los alumnos sin diferencias regionales ni sociales, no estaban adquiriendo los conocimientos básicos que se suponía debería impartirles la escuela, y los maestros a su vez no poseían los conocimientos necesarios para implementar la reforma del 63 (Kaessmann, 1966).

Las implicaciones en términos de política educativa durante el período se resumen así (Mojica, 1974).

1. Se requiere una transformación radical de la formación del normalista y un esfuerzo masivo de capacitación permanente del magisterio en servicio.

2. Las escuelas carecen de los materiales mínimos de enseñanza que permitan aprovechar los esfuerzos que se adelantan en la formación y capacitación del magisterio.

3. Se requiere un replanteamiento radical de la política de asignación de recursos diferentes al de los últimos años que ha orientado todos sus esfuerzos a la construcción de escuelas y la ampliación de plazas del magisterio.

La mejora cualitativa se centra entonces en dos instrumentos básicos: la capacitación de los docentes en servicio y el suministro de materiales didácticos para ser utilizados en el proceso de enseñanza-aprendizaje.

Dentro de este contexto el gobierno colombiano firma, el 25 de noviembre de 1968 un convenio con la República Federal de Alemania, conocido como "Segundo Convenio Adicional", el cual es retroactivo al lo. de octubre de 1967. Este convenio no surge sólo de conversaciones ni comunicados entre las partes, sino que cuenta con una experiencia previa. El señor Walter Kaessmann, director del Proyecto, venía vinculado al Ministerio de Educación de Colombia desde 1965 en calidad de asesor, época en la que había participado en actividades como el diagnóstico de la situación de la enseñanza de la matemática y el lenguaje, el diseño del Decreto 150 de 1967, la elaboración de un mapa de los planteles de primaria, el diseño y -aplicación de pruebas a maestros de primaria y, en general, había colaborado en la selección de problemas concretos de organización y planeación a nivel departamental. Anteriormente el señor Kaessmann había estado vinculado como docente de un colegio de la capital, época durante la cual había solicitado 
la introducción de un libro de matemáticas (1963) adaptado a los 5 años de la escuela primaria colombiana.

Son estas experiencias y prácticas las que de otra parte encauzan el convenio hacia la producción de materiales didácticos que constituyeran una ayuda para el maestro. Las encuestas habían demostrado que este era el punto débil de la educación primaria en Colombia. Refiriéndose a la enseñanza de la matemática por los maestros de 4o. año, anotaba el señor Kaessmann: "No superan los conocimientos de la materia en tal grado que les permita desarrollar la enseñanza didácticamente orientada... cada operación se desarrolla generalmente en la misma forma como lo recuerda el maestro desde su vida escolar. Por eso enseña, por ejemplo, la regla de tres en primer término como proporción porque es para el maestro la única forma que sabe" (Kaessmann, 1966).

El convenio suscrito trata entonces de aportar una solución a los problemas enunciados dentro de los siguientes términos: "Las partes contratantes colaborarán en el desarrollo de la enseñanza primaria en la República de Colombia mediante medidas de reforma en los sectores del perfeccionamiento del profesorado, de la organización práctica de la enseñanza y de los medios de la enseñanza" (Artículo lo.).

Este objetivo general se especifica en el ordinal 2o. en cinco actividades bien definidas:

a. Programación: Se trata de prestar ayuda para desarrollar los programas establecidos en virtud del Decreto 1710.

b. Producción de textos modelos.

c. Producción de material modelo.

d. Seminarios de capacitación en los diversos niveles.

e. Perfeccionamiento de Inspectores escolares.

En cuanto a la extensión del convenio vale la pena señalar que se pretendió desarrollar una acción inicial a través de escuelas de demostración, acción que debería ser continuada por el país huésped una vez que terminara el plazo del convenio, previsto inicialmente por un período de 5 años. Resumiendo, puede decirse que los objetivos de la Misión Pedagógica Alemana se concretaron en los aspectos didácticos y pedagógicos que tenían que ver con el acto de enseñanza-aprendizaje, lo cual debería cumplirse dentro de los lineamientos del Decreto 1710 de 1963.

Para el cumplimiento de los objetivos estipulados en el convenio el Gobierno Alemán se comprometió a enviar expertos alemanes (4 en un principio, adicionando 3 en 1971), a prestar ayuda financiera con destino al material didáctico y a los textos, y a suministrar un equipo sencillo de imprenta.

Por su parte el Gobierno colombiano se comprometió a constituir una comisión integrada por expertos alemanes y técnicos colombianos para el planeamiento y realización de las medidas de reforma. Asimismo era su competencia examinar la idoneidad de los planes, libros y materiales de enseñanza recomendados por la Misión. Finalmente, debería proveer a las escuelas con los medios elaborados y garantizar la debida utilización y administración del material. 


\section{Organización e implementación}

El análisis de la implementación de un programa es tal vez de los tópicos más complejos dentro del proceso de evaluación. En primer lugar porque factores políticos y burocráticos interactúan con factores técnicos, y en segundo, por la presencia de diversos estamentos dentro de la organización, desde el nivel decisorio hasta los receptores del programa, cuyo concurso impide u obstaculiza el logro de las metas trazadas al inicio del proyecto. La implementación (Williams, 1978), el estadio entre la decisión y la operación, empieza con el desarrollo de las guías generales del programa o el diseño de sus especificaciones, se mueve hacia lo que puede ser un largo camino de trabajo entre un centenar de problemas técnicos, administrativos e institucionales que enfrenta la nueva actividad; y termina cuando la actividad ha producido sus resultados y se pueden evaluar. Este proceso en el caso de la Misión Pedagógica Alemana se puede dividir en cuatro etapas: una primera de preparación que va de 1965 a 1968; una segunda de configuración y planeamiento, de 1968 a 1972; la tercera de expansión que cubre de 1973 a 1975; y la etapa de finalización y traspaso a las autoridades colombianas entre 1976 y 1978.

La etapa de preparación que termina con la firma del "Segundo Convenio Adicional" sienta las bases, como se mencionó anteriormente, para lo que habría de ser el objetivo y las actividades de la Misión: la reforma interna de la educación primaria a través del desarrollo de materiales y ayudas para el profesor.

En la etapa de Configuración y Planeamiento el trabajo se concentra en la programación, experimentación y producción de este material. Para este fin se confirma una comisión integrada por expertos alemanes y colombianos, cuya composición puede apreciarse en el Cuadro 1.

Los expertos colombianos eran técnicos de la enseñanza primaria con una gran trayectoria dentro del sector. Los alemanes, tenían un nivel académico superior pues las normales alemanas, donde se habían formado, pueden equipararse a las facultades de educación.

Esta comisión se distribuyó en grupos de trabajo por áreas (matemáticas, lenguaje, estudios sociales, ciencias naturales, educación física, música, dibujo, manualidades) para el desarrollo de los materiales. Los grupos eran autónomos para tomar las decisiones de carácter pedagógico y didáctico, "adoptaron un sistema de disciplina interna que le permitía a cada quien expresar con toda libertad sus ideas y utilizar tanto sus conocimientos como sus experiencias docentes" (Ramírez, 1978). En estos grupos de trabajo se dio una transferencia tecnológica para el desarrollo de los materiales, que fue tal vez mayor en trabajos manuales, música o educación física, áreas muy deficientes dentro del programa nacional. 


\section{CUADRO 1}

\begin{tabular}{|c|c|c|c|}
\hline Año & $\begin{array}{c}\text { Técnicos } \\
\text { Colombianos }\end{array}$ & $\begin{array}{c}\text { Expertos } \\
\text { Alemanes }\end{array}$ & Total \\
\hline 1965 & 4 & 1 & 5 \\
\hline 1966 & 12 & 1 & 13 \\
\hline 1967 & 16 & 1 & 17 \\
\hline 1968 & 18 & 3 & 21 \\
\hline 1969 & 15 & 3 & 18 \\
\hline 1970 & 15 & 5 & 20 \\
\hline 1971 & 14 & 6 & 20 \\
\hline 1972 & 17 & 7 & 24 \\
\hline 1973 & 31 & 7 & 38 \\
\hline 1974 & 36 & 7 & 43 \\
\hline 1975 & 22 & 7 & 29 \\
\hline
\end{tabular}

El grupo estaba bajo la dirección de un experto alemán, el señor Walter Kaessmann, quien de acuerdo con lo estipulado en el convenio actuaba en lo administrativo y en lo pedagógico; lo primero tenía que ver con manejo de fondos, contratos con las empresas productoras y vigilancia de los contratos. El señor Kaessmann además de contar con una gran habilidad política y administrativa, le daba al proyecto algunas características muy peculiares, especialmente una gran autonomía (Arnold-Bergstraesser, 1974).

Organizativamente (hasta 1973) el proyecto dependía directamente de la oficina del Ministro, lo cual lo ponía en contacto con el nivel decisorio "evadiendo las instituciones formalmente competentes y las instancias intermedias de la burocracia" (Arnold Bergstraesser, 1974). De otra parte, la amplia autonomía financiera y la libertad que le otorgó el BMZ* reforzaban esta actitud, por lo cual prácticamente el grupo respondía a un plan interno de trabajo previamente aprobado por las autoridades colombianas.

Si bien esta estructura le dio agilidad para la ejecución de sus actividades, también se convirtió en un obstáculo para la continuidad del programa, tal como se verá más adelante. El proyecto a finales de 1972, había terminado la producción de las guías y los libros de matemáticas para los maestros de 1o. y 20. curso, y había iniciado la producción de los otros libros y de los materiales didácticos. Los Cuadro A1 a A5 resumen los materiales impresos durante esta primera fase. La prórroga del convenio se hacía entonces necesaria para que el programa no quedara inconcluso.

Al finalizar esta segunda etapa y tal vez como una reacción a la autonomía institucional de la Misión Pedagógica Alemana se presenta una confrontación de fuerzas entre algunos mandos del MEN y la dirección de la Misión: como consecuencia de la reforma administrativa de 1968 se habían creado algunos institutos públicos del sector educativo ocasionando una desarticulación programática. Varios institutos y unidades administrativas luchaban entre sí por una participación mayor en el presupuesto nacional ante el cual cada uno presentaba su propio proyecto.

De otra parte, las agencias internacionales y los países que prestaban apoyo financiero al país habían logrado imponer un sistema que consistía en apoyar económicamente acciones sueltas. Los representantes de estas agencias o países declaraban prioritarias

\footnotetext{
* Ministerio Federal Alemán para la Cooperación Internacional. 
ciertas acciones y las sugerían a los gerentes o directores de los institutos o jefes de unidades administrativas del Ministerio.

Ante esta situación se elaboró el Plan de Desarrollo del Sector Educativo y se hizo un esfuerzo para establecer una política de cooperación técnica. Esta última fue fijada por el Departamento Nacional de Planeación, cuyas normas más importantes son resumidas así (Ceballos):

— Se cambia el concepto de la asistencia técnica por el de "Cooperación técnica internacional".

— La cooperación internacional debe ajustarse al plan nacional de desarrollo. La cooperación técnica y la cooperación financiera se manejan por separado.

- La iniciativa para los proyectos de cooperación técnica internacional debe partir de las entidades nacionales y no de las agencias internacionales.

-- La entidad receptora debe disponer de suficientes recursos humanos y físicos para garantizar el desarrollo normal de los programas.

- La contrapartida del presupuesto nacional debe asegurarse, conforme al plan de desarrollo nacional, ante el propio gobierno; y éste, según el orden de prioridades del plan, la incluirá en la respectiva vigencia fiscal.

-El gobierno procurará conformar un plan integrado de cooperación técnica internacional, en el cual tendrá primacía la cooperación multilateral. Dentro del plan, la cooperación bilateral no tendrá importancia menor, pero en el diseño del esquema nacional tendrá carácter de complementariedad.

Los resultados de esta política se reflejan claramente en la Resolución 8304 del 14 de diciembre de 1972, cuya parte motivadora dice así:

"Considerando que todas las actividades de cooperación técnica internacional del sector educativo deben ceñirse al plan nacional de desarrollo sectorial para lo cual es preciso incorporarlas por afinidades a los proyectos que comprende dicho plan,

-Que el artículo 5o. de la Resolución 1197 de 1971 dispone: "Los proyectos de las entidades del sector que se realicen con cooperación técnica internacional tendrán un director colombiano, responsable de la ejecución del proyecto...".

-Que esa designación es necesaria para que Colombia ejerza las facultades y asuma plenamente las responsabilidades que le corresponden como cooperante en la realización de tales proyectos...".

"Resuelve, incorporar al proyecto No. 2 del plan nacional de desarrollo del sector educativo ("Capacitación y perfeccionamiento del personal docente en servicio"), cuyo ejecutor es el Ministerio de Educación a través del Centro Nacional de Perfeccionamiento Educativo (CENAPER), las actividades de cooperación técnica que actualmente realiza la República Federal de Alemania a través de la Misión Pedagógica Alemana". 
Esta resolución implicaba una nueva interpretación del Segundo Convenio adicional ya que las funciones del director de la Misión quedarían reducidas a la dirección interna del grupo alemán. Sin embargo, el 24 de enero de 1973, el Ministerio produce una nueva Resolución, la No. 0280, que modifica el artículo 10. de la No. 8304, estableciéndose lo siguiente:

- Se confirma el carácter de asesora que tiene la Misión con respecto al cuerpo de la educación primaria en los términos establecidos en el convenio firmado entre Colombia y Alemania Federal (Art. 1).

—Se adscribe la Misión Alemana a la Oficina de Planeación del Ministerio (Art. 2).

- La sede de la Misión para los efectos de la administración estará ubicada en el Ministerio (Art. 3).

-En su carácter de asesora, la Misión prestará sus servicios al CENAPER y al ICOLPE en cuanto hace relación a la capacitación de personal en servicio, procesamiento de ayudas didácticas e investigaciones pedagógicas y programación (Art. 4).

El desenlace fue entonces el afianzamiento de la autonomía de la Misión Pedagógica Alemana, situación que da base a la firma del convenio de prórroga.

En esta situación se inicia la fase de expansión, aumentándose tanto el personal colombiano como el alemán (Ver Cuadro 1) y reforzándose el programa de cooperación técnica con otras iniciativas tendientes a solidificar la acción iniciada, como fueron la instalación de una imprenta y la creación de un centro audiovisual y del Centro Modelo de Cali. En esta etapa se dio cabal cumplimiento a los planes de producción de materiales enunciados en el convenio de 1968, terminándose la impresión de las guías de 3o., 4o. y 5o., de los libros para el profesor y de todos los materiales didácticos.

Durante esta fase se distribuyen los materiales al magisterio y a las instituciones encargadas de la capacitación del magisterio. Los seminarios de actualización a maestros fueron el instrumento para obtener las guías, mediante lo cual se buscaba también asegurar la efectividad de la Misión (Ulmer, 1978).

Para la ejecución del programa de capacitación se inició un trabajo con los supervisores departamentales, con el fin de aprovechar sus servicios. Sin embargo, por motivos laborales, como era el recargo de trabajo, esta colaboración falló. Al no poderse concretar esta ayuda se hizo otro esfuerzo consistente en el nombramiento de "multiplicadores" cuyo mecanismo se explicitaba en una comunicación del señor Viceministro de Educación, Hernando Ochoa Núñez y enviada en febrero de 1974 a los delegados del FER. La labor de capacitación fluctuó de acuerdo con la posibilidad que tuvo cada departamento, de nombrar maestros en comisión para esta actividad. Se llegó a destinar un total de 444 multiplicadores en todo el país, que atendieron un promedio de 200 maestros cada uno.

A finales de esta fase, en 1974, se realiza una evaluación de la Misión por parte del Gobierno Alemán para lo cual se contratan cuatro evaluadores del Instituto Arnold Bergstrasser, quienes a su vez se asesoraron en su labor por 2 profesionales colombianos. Dentro de los resultados se menciona el alcance de la producción de 
materiales "tanto desde el punto de vista del volumen como de la calidad" y la elevada cuota de realización de los distintos programas, atribuyendo los resultados "a la fuerte motivación y disponibilidad para el trabajo de los distintos miembros del proyecto, así como a la habilidad política y organizativa del director del proyecto". Sin embargo, se le critica el que "se renunciara al intento de cambiar las estructuras de la escuela primaria.., concentrándose más bien en las diversas tareas que se relacionaban con el mejoramiento de la calidad pedagógica - didáctica de la escuela primaria". Se pone de manifiesto por el grupo evaluador la falta de una labor crítica frente a las valoraciones socio-políticas y las actitudes transmitidas a través de la escuela, cuya "re-novación no fue vista como una tarea de la Misión Pedagógica Alemana". Otra limitación, la refieren a la "renuncia de la "cientificidad" de sus actividades" ya que no se hizo ningún esfuerzo de sustentar el trabajo con investigaciones básicas o evaluaciones. Finalmente, lamentan el que la "relativa autonomía institucional" impidiera la colombianización del proyecto (Arnold Bergstrasser, 1974).

Con respecto a la prórroga del convenio los evaluadores del Instituto Arnold Bergstrasser recomiendan:

- Preparar el retiro definitivo de la ayuda Alemana mediante una etapa de traspaso de 3 años.

— La necesidad de que Colombia participe más activamente.

- La necesidad de que un alemán codirigiera la producción de materiales, una de cuyas tareas sería la de revisar con especialistas colombianos los programas desarrollados por la Misión Pedagógica Alemana.

- Reducir el volumen de la ayuda económica por parte de Alemania para la producción de medios de aprendizaje.

De acuerdo con lo recomendado se inicia en 1976 la etapa de traspaso bajo la dirección del señor. Heino Ulmer, época en que se redujo la acción de la Misión a la cooperación financiera y a la realización de seminarios para el perfeccionamiento de los maestros. Como puede observarse en los Cuadros Al a AS para esta época ya se había terminado la producción de todo el material, dándose énfasis a la impresión, distribución y realización de seminarios. En 1976, se efectuaron seminarios nacionales en dibujo, obras manuales y educación musical.

Según el informe final (Ulmer, 1974) uno de los alcances de la Misión Pedagógica Alemana fue la asesoría al MEN sobre la creación de nuevas divisiones para el desarrollo del currículo y elaboración de material didáctico. Como resultado de la actividad asesora, al reformarse el Ministerio a través del Decreto 088 del 22 de enero de 1976, se crea la "Dirección General de Capacitación y Perfeccionamiento Docente, Currículo y Medios Educativos", para asumir el trabajo que la Misión había venido realizando en favor de la escuela primaria. Este decreto y el Decreto 1419 de 1978 reestructuran el sistema educativo, creando la educación pre-escolar y educación básica que comprende la educación básica primaria de 5 años y básica secundaria de 4 años, otorgándole a la Dirección General de Currículo la responsabilidad de elaborar los planes y programas de la nueva reforma educativa. 
En 1977 empieza a funcionar esta dependencia bajo la dirección de Pilar Santamaría de Reyes, quien a través de una comunicación el 29 de agosto del mismo año le solicita un informe al señor Ulmer y designa una comisión de tres personas para que "elaboren una propuesta de trabajo para la coordinación de la ejecución del proyecto hasta la fecha de terminación, con el objeto de que esta Dirección... pueda asumir la responsabilidad de ejecución de este proyecto de cooperación técnica internacional".

Como resultado de esta solicitud el director del proyecto le informa sobre las actividades realizadas por la Misión y le sugiere "estudiar la posibilidad de solicitar colaboración económica por parte del Gobierno alemán para la producción de un ampliado y adecuado paquete de material didáctico con destino al nuevo currículo de la educación básica primaria y secundaria".

Del análisis de las varias comunicaciones entre las partes puede inferirse que no hubo acuerdo para que la Dirección de Capacitación pudiera asumir la responsabilidad de la ejecución del Proyecto. Así en abril de 1978, en una carta al señor Ulmer se le informa que "aproximadamente en el mes de junio de este año estarán listos para impresión los programas y unidades integrados de 10., 2o., y 3o., grados de básica primaria producidos por la División de Diseño y Programación Curricular de Educación formal... Con respecto a este punto y de acuerdo con lo decidido en el plan de operaciones 1978 y ratificado durante las visitas de funcionarios de la G.T.Z., el costo y los recursos para la producción de guías de lo., 2o., y 3o. se haría con cargo a este proyecto y remplazarían a las producidas en años anteriores ${ }^{1}$.

Desde el punto de vista operativo y estratégico puede decirse que no hubo un empalme entre las dos reformas al no producirse un seguimiento y evaluación de los materiales producidos por la MPA, ni una coordinación efectiva entre el grupo que colaboraba con esta última y las oficinas encargadas del diseño curricular. Esta falta de "institucionalización" del proyecto pudo haber significado si no la pérdida, al menos la minimización de diez años de trabajo de un grupo de colaboradores colombianos que trabajó bajo la asesoría alemana.

\section{Los resultados de la Misión}

De acuerdo con lo expresado en los antecedentes del programa, el objetivo perseguido por el Gobierno colombiano al contratar la asesoría alemana era el de lograr una mejora en la calidad de la enseñanza primaria, o como se le denominó, una reforma interna de la educación primaria. Para alcanzar este fin último se previó una serie de instrumentos que se constituyeron en la condición necesaria para cumplir con el objetivo deseado y que quedaron consolidados en el convenio, a saber: el desarrollo de los programas del 1710 y su entrega a los maestros en forma de guías didácticas; la elaboración de textos y ayudas didácticas; y la capacitación y actualización a los maestros sobre el material y la metodología de enseñanza necesaria para su uso. (Diagrama 2).

Estos resultados pueden ser desagregados aún más en una serie de pasos cuyo cumplimiento garantizaría no sólo el alcance del objetivo final sino de objetivos intermedios, tal como se aprecia en el diagrama 3. La base, constituye la efectividad de la Misión en desarrollar los planes y programas estipulados en la reforma del 1710; un segundo nivel lo establece la capacidad para reproducir masivamente este material y, como tercer nivel, distribuirlo y garantizar su recepción por los usuarios potenciales

\footnotetext{
${ }^{1}$ El subrayado es nuestro. 
(escuelas, maestros, alumnos). Una vez que el material esté en manos de los usuarios la efectividad está dada por la comprensión que el maestro tenga del material y su uso efectivo en el aula de clase; sólo así tendrá lugar la mejora de la calidad educativa. Este último paso, como ya se ha dicho no fue considerado objetivo de la presente evaluación, por lo cual sólo se presentarán los resultados de los niveles 1 a 5.

\section{a) Desarrollo y adaptación de los programas del 1710.}

Como se mencionó anteriormente, los resultados de las pruebas aplicadas a 60.000 estudiantes de la escuela primaria y 2.800 maestros demostraron que estos últimos carecían de los elementos suficientes para cumplir la reforma del 63. Como medio para implementar la reforma educativa del 63 se escogió entonces la elaboración de guías y textos para los maestros, textos para los alumnos y ayudas educativas para utilizar en el aula de clase.

Las guías son un desarrollo de los planes estipulados en el Decreto 1710 mediante la organización de los programas, el suministro de pautas metodológicas, y el desarrollo y ampliación de los contenidos. Su objetivo es el de dotar al maestro de un instrumento que le permita desarrollar el programa del curso indicándole la metodología para su enseñanza y sugiriéndole actividades prácticas complementarias, con el fin de alcanzar la deseada mejora cualitativa del proceso enseñanza-aprendizaje.

Las guías de 10. a 3er. curso constan de tres volúmenes que contienen la parcelación, el desarrollo y los anexos. La primera da una visión secuencial de los temas a tratar en cada semana; la segunda presenta las actividades a desarrollar de acuerdo con la parcelación temática para cumplir los objetivos propuestos; en los anexos se amplían los contenidos y se presentan las indicaciones metodológicas necesarias para tratar los temas. Para los cursos 4o. y 5o. cada guía desarrolla un área o asignatura particular. 


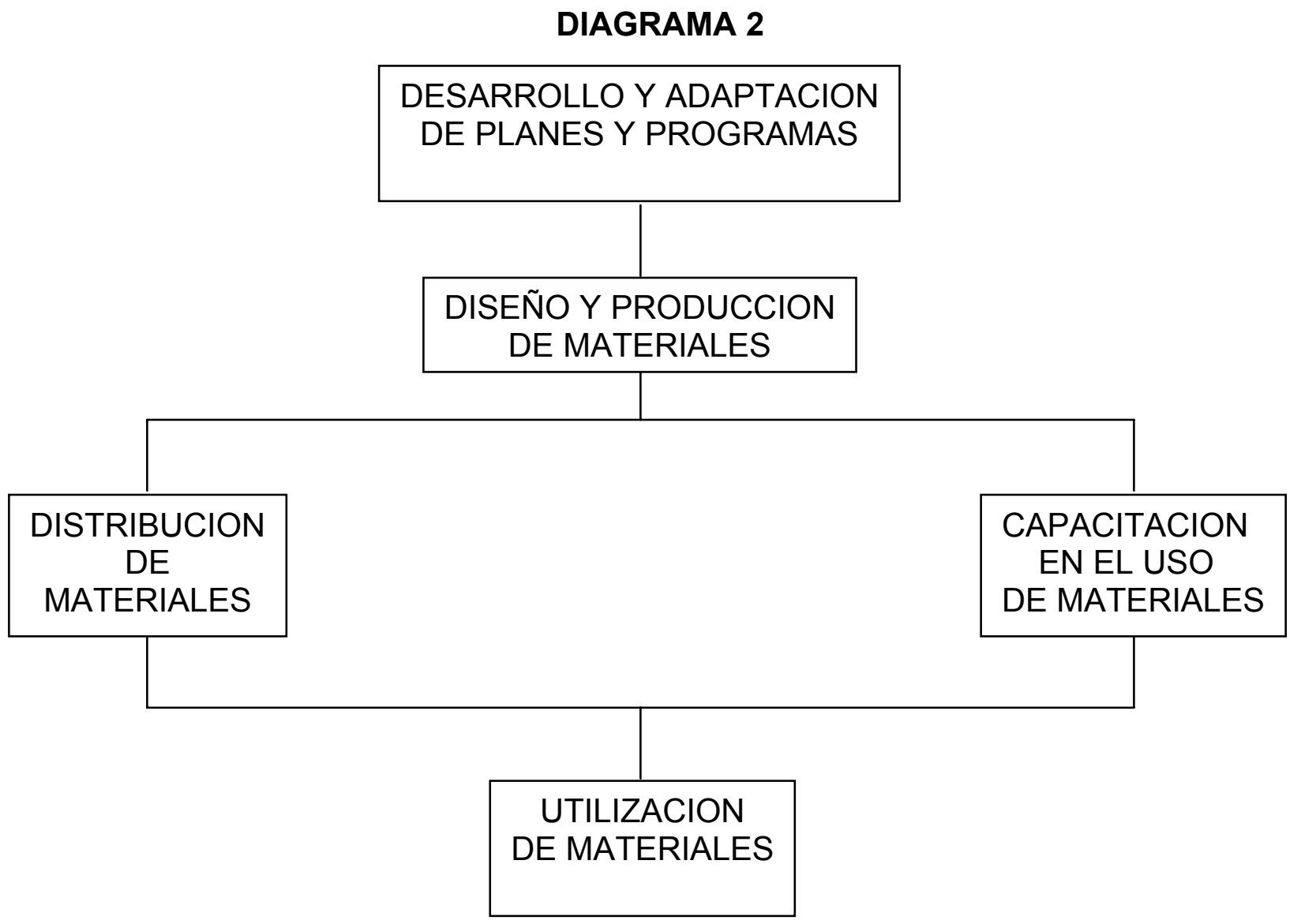

DIAGRAMA 3

6. Mejoramiento de la calidad educativa.

5. Uso del material.

4. Comprensión del material y adaptabilidad.

3. Recepción del material por los usuarios.

2. Producción masiva del material.

1. Desarrollo curricular a través de guías, textos y materiales. 
Desde el punto de vista metodológico las guías superan los programas inicialmente entregados a los maestros, los cuales indicaban lo que el maestro debería enseñar pero no cómo hacerlo.

En cuanto a la organización de los contenidos las guías de 1o. y 20. curso son "globalizadas" y la parcelación se hace semanalmente, correlacionando algunos temas afines. La guía de 3er grado es una forma de transición entre la enseñanza globalizada y la epocal del 4o. y 5o. grado, donde se presenta cada área de conocimiento en forma separada y la parcelación se realiza por unidades y por quimestres. En estos últimos grados las guías presentan algunas modificaciones respecto al Decreto 1710: En el área de ciencias naturales se introducen nociones fundamentales de física y química, en biología se organiza el contenido bajo un enfoque ecológico.

Adicionalmente a las guías se ofrecen varios textos que apoyan la labor del maestro en las áreas de educación física, manualidades y música. Para la primera se elaboraron dos tomos de "Educación Física en la Escuela Primaria" que cubren del primero al tercer grado y del cuarto al quinto respectivamente. Estos libros presentan en forma amplia y completa las actividades que el maestro puede ejecutar durante el año escolar y se correlacionan temas con otras áreas del conocimiento. En educación musical se ofrecen dos cancioneros "Viva la Música" con cantos infantiles, el primero y con aires juveniles de diversas regiones tanto del país como del extranjero, el segundo. Para el desarrollo de manualidades se elaboraron dos volúmenes "La Clase Alegre" y "Otra Clase Alegre" con sugerencias para el desarrollo de actividades como plegados, títeres, adornos, montajes, siluetas, trabajos de tejidos y otras.

El grupo trabajó también en la producción de dos series de libros de texto para los alumnos, "Calculemos" y "Vamos a Leer": El primero para los grados de 10. a 5o.; el segundo, únicamente para el primer curso.

Parte de la estrategia de producción de materiales consistió en dotar a las escuelas de un conjunto de ayudas educativas para las áreas de lectura, matemáticas, geografía, historia, biología y trabajos manuales. Los materiales elaborados fueron los siguientes:

Sellomat: Son sellos diseñados básicamente para la etapa del aprestamiento. Mediante ejercicios los alumnos pueden realizar ejercicios prácticos sobre conjuntos, colorear y visualizar los elementos de dichos conjuntos.

Fracciograf: Está relacionado con la conceptualización de los números fraccionarios.

Metro Cúbico: Desarmable, constituido por 12 varillas plásticas que se unen entre sí, para conceptualizar mediante la visualización, el metro cúbico y sus submúltiplos.

Decímetro Cúbico: Son dados, palitos y placas de plástico para explicar el decímetro cúbico y su descomposición en centímetros cúbicos.

Figuras Geométricas: Este material consta de diferentes figuras: prismas, pirámides, un cono, un cilindro y dos recipientes de medición, de un litro y un decilitro.

Fichero: Material auxiliar para la enseñanza de la lectura; en el manejo didáctico del fichero se recomienda combinarlo con el Lotelect y la cartilla "Vamos a Leer 
Sellolect: Sellos de lectura. Material diseñado para los grados de lo. y 2o. de primaria. Consta de tres sellos con su correspondiente almohadilla y una guía impresa para el maestro. Este material se complementa con el fichero, el Lotelect y la cartilla "Vamos a Leer". Los sellos contienen palabras y letras impresas que al reproducirlas sobre un papel en blanco, los alumnos pueden recortar y formar con ellas, palabras y oraciones.

Juegos de mapas: Serie formada por seis mapas impresos que contiene: Un mapamundi político, un mapamundi físico, un mapa físico de Suramérica, un mapa político de Suramérica, un mapa físico de Colombia, un mapa político de Colombia.

Al dorso de cada mapa están impresos datos estadísticos e informativos complementarios. Este juego de mapas se ofrece a maestros y alumnos a partir del 3er. grado hasta el 5o. de primaria. Es importante anotar que este material sirve también para la enseñanza media.

Globo terráqueo: Elaborado en material plástico inflable, tiene una doble funcionalidad, para la enseñanza de la geografía a partir de segundo de primaria, y para ser utilizado en juegos escolares.

Sellos de geografía: Que constan de: Un sello de caucho con el mapa de Colombia, un sello de caucho con el mapa del departamento, un sello de caucho con el mapa de América del Sur.

Este material es utilizable de acuerdo a los grados y según la programación y desarrollo de las guías preparadas para los maestros de enseñanza primaria.

Frisos de historia: Está conformado por seis frisos o láminas, con una secuenciación cronológica según los acontecimientos y sucesos históricos más importantes en Colombia. En el dorso de los frisos o láminas está impreso un sistema de evaluación para ser utilizado por el maestro. Anexo a este material se elaboró una guía que amplía, explica y complementa el contenido de los frisos.

Láminas de biología humana: Consta de una serie de doce láminas en total, destinadas tanto a los maestros como a los alumnos de los grados 4o. y 5o. de primaria. Además existe una guía que orienta la labor del maestro al mismo tiempo que amplía y complementa didácticamente los contenidos de cada una de las láminas.

Desde el punto de vista del desarrollo programático y curricular puede decirse que el trabajo efectuado constituye un modelo sin precedentes dentro de la historia de ha educación en Colombia. A través del desarrollo de los materiales se profundizan algunas áreas como ha física y la química para 4o. y 5o. grado, y se imprime un carácter diferente e innovativo a la música, trabajos manuales y educación física, áreas que tenían un nivel incipiente en el país. Se introduce y se realiza la idea de unificar y suministrar textos subsidiados a los alumnos de la educación oficial. Con este fin se proyectan los textos de matemáticas, "Calculemos", y de lectura, "Vamos a Leer", los cuales serían entregados a los alumnos a un precio muy económico. El primero de ellos, "Calculemos", fue criticado por ser una traducción del alemán pese a que había sido adaptado y probado por profesores colombianos; esta publicación se suspendió en 1975. De la otra, únicamente se publicó el Volumen 1, quedando listos los materiales del libro 2, no obstante ser un trabajo netamente colombiano ya que el único alemán que trabajaba allí fue retirado del grupo redactor. El fracaso de esta estrategia no se puede atribuir sólo a has razones pedagógicas de los textos, sino también a la falta de apoyo del nivel decisorio del 
Ministerio respecto a la entrega de textos para los alumnos. Así por ejemplo el Gobierno del Frente Nacional puso especial interés en la cartilla "Vamos a Leer", la cual fue financiada casi en su totalidad por el Gobierno, mientras que los manuscritos de la segunda que estaban listos nunca se imprimieron.

\section{b) Producción de materiales}

Los Cuadros A-1 a A-5 hacen una compilación de la producción de materiales presentados en los informes finales de Kaessmann y Ulmer. De cada tipo de material se realizaron varias ediciones o reproducciones a medida que iba avanzando la Misión. La guía para el maestro de lo. fue actualizada en 1975, la de segundo en 1976; en cuanto a las guías de 3o. grado, se hallaban en proceso de actualización en 1978 al clausurarse la Misión; posteriormente alcanzó a salir una edición resumida de esta guía. En total se imprimieron cerca de 7 millones de libros correspondientes a los 25 prototipos elaborados; de las 19 ayudas educativas que se diseñaron se produjo un promedio de 15.000 de cada una.

Con eh fin de poder evaluar los alcances de la Misión en términos del cubrimiento de las necesidades totales del sistema educativo colombiano se efectuó una comparación entre la producción y el número de posibles usuarios de estos materiales, a lo que se denomina "cobertura potencial".

Eh Cuadro 2 presenta los resultados para las guías; el cálculo de la cobertura se hizo con base en el total de docentes vinculados al sector oficial en cada uno de los cursos para los que las guías iban dirigidas. Como puede observarse en todos los casos se produjeron más del doble de ejemplares, lo cual haría suponer que la producción alcanzaba para cubrir el total de maestros dejando un margen bastante amplio para remplazar en caso de incremento en el número de docentes o de pérdida por deterioro. Las guías "Calculemos" para el uso del maestro fueron producidas en menor cantidad, sin embargo, el índice de cobertura es mayor de uno, excepto para los cursos 40. y 5o. (Ver Cuadro 3).

\section{CUADRO 2}

\begin{tabular}{|c|c|c|c|}
\hline \multicolumn{4}{|c|}{$\begin{array}{l}\text { CUBRIMIENTO DE LAS GUIAS PARA EL MAESTRO CON BASE } \\
\text { EN EL NUMERO DE DOCENTES }\end{array}$} \\
\hline Material & $\begin{array}{c}\text { Docentes' } \\
\text { Sector Oficial }\end{array}$ & Producción & Cobertura \\
\hline Guía de lo. & 36.308 & 90.000 & 2.48 \\
\hline Guíade2o. & 23.689 & 80.000 & 3.38 \\
\hline Guía de 30. & 19.293 & 50.000 & 2.59 \\
\hline Guía de 4o. & 13.681 & 40.000 & 2.92 \\
\hline Guía de So. & 11.258 & 30.000 & 2.66 \\
\hline
\end{tabular}

Docentes del año 1975

El índice correspondiente a los textos para alumnos (Cuadro 3) se calculó con base en la matrícula escolar de cada curso. El texto "Vamos a Leer" tuvo un tiraje que doblaba a los alumnos de primer año, los demás tienen una cobertura de casi ciento por ciento con excepción de "Calculemos" 40. y 5o. (Cuadros 4 y 5).

La cobertura de los demás libros para el maestro se calculó con base en el número de establecimientos, al igual que la de los materiales didácticos, ya que se supuso que un 
juego de éstos bastaba para suplir las necesidades de todo el plantel, independientemente del número de alumnos o docentes. Para los primeros la cobertura duplicaba o triplicaba el número de establecimientos (Cuadro 4), para los materiales educativos la cobertura es de .5 en promedio, es decir que hubieran alcanzado para la mitad de establecimientos.

\section{CUADRO 3}

\begin{tabular}{|cccc|}
\hline \multicolumn{4}{|c|}{ CUBRIMIENTO DE LAS GUIAS CALCULEMOS PARA EL MAESTRO } \\
& CON BASE EN EL NUMERO DE DOCENTES & \\
\hline Material & $\begin{array}{c}\text { Docentes } \\
\text { Sector Oficial }\end{array}$ & Producción & Cobertura \\
\hline Guía Calculemos 1 & 36.308 & 58.000 & 1.60 \\
Guía Calculemos 2 & 23.689 & 41.000 & 1.73 \\
Guía Calculemos 3 & 19.293 & 27.000 & 1.40 \\
Guía Calculemos 4 & 13.681 & 8.000 & 0.58 \\
Guía Calculemos 5 & 11.258 & 10.000 & 0.89 \\
\hline
\end{tabular}

Docentes del año 1975.

\section{c) Distribución de los materiales}

Según la letra del Segundo Convenio Adicional, la distribución de los materiales didácticos producidos por la Misión Pedagógica Alemana, correspondía exclusivamente a la República de Colombia. El artículo 7 del citado convenio decía en su inciso 40.

\section{CUADRO 4}

\section{CUBRIMIENTO DE CALCULEMOS Y VAMOS A LEER PARA EL} ALUMNO CON BASE EN EL NUMERO DE ALUMNOS DE 1975

\begin{tabular}{|lccc|}
\hline Material & $\begin{array}{c}\text { Alumnos } \\
\text { Sector Oficial } \\
\text { por cursos }\end{array}$ & Producción & Cobertura \\
\hline Calculemos de lo. & 1.152 .874 & 1.370 .000 & 1.19 \\
Calculemos de 2o. & 753.317 & 1.055 .000 & 1.40 \\
Calculemos de 3o. & 614.269 & 770.000 & 1.25 \\
Calculemos de 4o. & 439.047 & 385.000 & 0.88 \\
Calculemos de 5o. & 359.070 & 250.000 & 0.70 \\
Vamos a Leer & 1.152 .874 & 2.400 .000 & 2.08 \\
\hline
\end{tabular}




\begin{tabular}{|lccc|}
\hline \multicolumn{4}{|c|}{$\begin{array}{c}\text { CUBRIMIENTO DE LIBROS PARA EL MAESTRO CON BASE EN } \\
\text { EL NUMERO DE ESTABLECIMIENTOS OFICIALES DE 1977 }\end{array}$} \\
\hline \multicolumn{1}{|c}{ Material } & Establecimientos & Producción & Cobertura \\
\hline Viva la Música 1 & 29.763 & 114.000 & 3.83 \\
Viva la Música II & 29.763 & 39.000 & 1.31 \\
La Clase Alegre & 29.763 & 39.000 & 1.31 \\
Otra Clase Alegre & 29.763 & 39.000 & 1.31 \\
Educación Física 1 & 29.763 & 74.000 & 2.49 \\
Educación Física II & 29.763 & 66.000 & 2.22 \\
\hline
\end{tabular}

"El gobierno de la República de Colombia proveerá dentro de su competencia las escuelas primarias con los medios de enseñanza recomendados por la comisión mencionada en el inciso 2 y aprobadas por el Ministerio de Educación Nacional, siempre que el Gobierno de la República Federal de Alemania no corra con los gastos de acuerdo con el artículo 4".

Según lo anterior, los gastos de transporte que demandaba la distribución de los materiales, hasta colocarlos en las capitales de Departamento, Intendencia o Comisaría, los pagaba el Ministerio de Educación. Para agilizar las gestiones de traslado, la dirección de la Misión Pedagógica Alemana cancelaba los costos de transporte a las empresas transportadoras y luego pasaba la relación completa al Ministerio, el cual reintegraba el dinero a la Dirección de la Misión.

Además, la Misión Pedagógica Alemana controlaba la distribución inmediata a las escuelas, exigiendo los comprobantes de recibo por parte de éstas en las oficinas de la Misión.

Por regla general el material producido se distribuía por intermedio de las entidades educativas nacionales que funcionaban en las secretarías de Educación; tratándose de las Guías y de los libros para el maestro, éstos iban generalmente sin intervención del delegado inmediato del Ministerio, a los Centros Regionales (hoy Centros Experimentales Piloto) los cuales los entregaban a los maestros que hacían los cursos de perfeccionamiento docente.

Posteriormente, cuando los FER en virtud de acuerdo firmado entre las secretarías de Educación y el Ministerio del mismo ramo entraron a colaborar en la capacitación del magisterio con los maestros "multiplicadores" tuvieron el encargo de distribuir los materiales. Así lo establece la Resolución No. 12259 del 15 de diciembre de 1976, artículo 40.: "Los delegados del Ministerio de Educación ante los Fondos Educativos Regionales --FER- son responsables, conjuntamente con los jefes encargados de los programas de capacitación en cada Departamento, Intendencia, Comisaría y el Distrito Especial de Bogotá, del recibo, la guarda y la distribución material de los elementos didácticos a los cuales se refiere esta resolución. Tanto el delegado como el jefe del programa de capacitación de la respectiva entidad territorial quedan con la obligación de remitir al Ministerio de Educación Nacional el informe detallado sobre la distribución realizada, la cual se hará teniendo en cuenta las listas de escuelas y maestros que hubiesen recibido estos mismos elementos en ocasiones anteriores, para evitar duplicaciones en la distribución". 
Todos los materiales didácticos producidos por la Misión Pedagógica Alemana, se distribuyeron gratuitamente; sin embargo, los maestros que recibían las guías debían pagar un precio bajo, diez pesos; luego, la Resolución del Ministerio, No. 2289, del 7 de mayo de 1975 , estableció unos precios que oscilaban entre $\$ 20.00$ y $\$ 50.00$.

Para la distribución a los departamentos, se estableció una proporción la cual fue luego reducida para algunos, como se observa en el Cuadro 6.

Los segundos porcentajes fueron establecidos por la Resolución No. 12259 del 15 de diciembre de 1976, artículo 30.

A pesar de que la distribución contó con normas, mecanismos operativos y controles bien establecidos como se deduce por lo expuesto, en la práctica se presentaron algunas dificultades. El informe del Instituto Amold-Bergstraesser señala algunas de ellas como es el dar a los materiales un uso inadecuado (retener los libros, venta, etc.). "En general, no se trata de una pérdida durante la distribución, sino de serias dificultades de organización". (Amnold-Bergstraesser, 1974).

En ocasiones, las autoridades seccionales de educación no se atenían a las normas técnicas para la distribución del material; por ejemplo, se daba el caso de repartir las guías sin haber dado la capacitación previa a los maestros como se desprende de una carta dirigida por el señor Ulmer al doctor Ernesto Panesso Robledo, delegado regional de Medellín (Octubre 27 de 1975). Según testimonios de personas que en los diversos frentes y lugares tuvieron que ver con el reparto de materiales, no era raro constatar que la distribución se hacía con móviles de tipo político y electoral.

\section{CUADRO 6}

\begin{tabular}{|l|r|r|}
\hline & $\%$ & $\%$ \\
\hline Antioquia & 10 & 9 \\
\hline Atlántico & 3 & 2.5 \\
\hline Bolívar & 3 & 2.5 \\
\hline Boyacá & 4 & 4 \\
\hline Caldas & 3 & 2.5 \\
\hline Cauca & 3 & 2.5 \\
\hline Cesar & 1.5 & 1.5 \\
\hline Córdoba & 3 & 2 \\
\hline Cundinamarca & 8 & 7 \\
\hline Chocó & 1.5 & 1.5 \\
\hline Distrito Especial & 8 & 7 \\
\hline Guajira & 1.5 & 1.5 \\
\hline Huila & 3 & 2.5 \\
\hline Magdalena & 2.5 & 2.5 \\
\hline Meta & 2 & 2 \\
\hline Nariño & 3 & 2.5 \\
\hline Norte de Santander & 3 & 2.5 \\
\hline Quindío & 2 & 2 \\
\hline Risaralda & 2.5 & 6 \\
\hline Santander & 7 & \\
\hline
\end{tabular}




\begin{tabular}{|l|r|r|}
\hline Sucre & 1.5 & 1.5 \\
\hline Tolima & 5 & 4 \\
\hline Valle & 8 & 7 \\
\hline Territorios Nacionales & 3 & 2 \\
\hline
\end{tabular}

Al realizar las encuestas en septiembre de 1981, en Medellín, varias personas que actuaron en la distribución del material de la Misión Pedagógica Alemana aseguraron que no hubo coordinación entre las entidades que distribuyeron el material, lo cual creó un verdadero caos: en efecto, hubo escuelas que recibieron el material varias veces y por conductos diferentes. Se dio el caso de que las autoridades de un municipio distante de la capital antioqueña, llamaron telefónicamente a la Secretaría de Educación para que fueran a recoger un material que estaba tirado en la vía pública y no había dónde almacenarlo.

A fines del año pasado, todavía se distribuyeron por parte del Centro Experimental Piloto de Medellín, por intermedio de los jefes de núcleo, guías a maestros bachilleres recién nombrados para zonas de difícil acceso.

Dado que el material era destinado para algunas escuelas con carácter experimental, y que de hecho no se podía realizar la dotación de todas, hubiera sido más efectiva la acción si se hubiera hecho un plan más riguroso de distribución, en cooperación con las autoridades seccionales y se hubiera realizado un seguimiento de ese material, tanto desde el punto de vista administrativo como didáctico.

EI ICCE inició la adquisición y distribución de materiales pedagógicos a partir de 1976 y no las ha suspendido; el suministro se hace para zonas rurales y urbanas. La institución no produce ni innova materiales; sólo licita y distribuye a través de su División de Estudios; los responsables de esta División se reúnen periódicamente con técnicos del Ministerio de Educación para determinar cuáles materiales se van a reproducir.

Últimamente se está buscando la manera de hacer más económico el conjunto de material didáctico; al efecto se suprimieron además las cajas de madera en las que se guardaban los diversos sellos.

\section{d) Capacitación para el uso de los materiales}

Parte de la efectividad del trabajo emprendido por la misión estaba condicionada a la capacitación de los maestros a través de seminarios sobre los materiales y la metodología en ellos empleada. En total se realizaron 2.949 seminarios sobre las guías y otros seminarios específicos en las áreas de trabajos manuales y dibujo, música y educación física. Si se supone que en cada curso toman parte 40 maestros, hasta finales de 1975 habían tomado parte 172.560 maestros (Ver Cuadro 7).

De 1976 a 1978, o sea durante la etapa de traspaso, los seminarios para la divulgación y el uso adecuado del material didáctico repartido, se realizaron únicamente a través de los equipos departamentales de multiplicadores.

Para apoyar las medidas colombianas en las tres áreas de educación estética, la República Federal de Alemania prometió enviar anualmente dos expertos por un corto plazo; en cumplimiento de esta promesa, en 1976 se realizaron seminarios nacionales en obras manuales y dibujo y en música bajo la participación de los señores Peter Hoffmann 
y Walter Dahl; en 1977 los dos expertos alemanes tuvieron que cancelar su participación con poca anticipación; en 1978 se prescindió por parte de Colombia del envío de los dos expertos alemanes, pues "en razón del avance en que se encuentra el diseño curricular para educación básica y las actividades desarrolladas por el personal nacional, no se hace necesaria la asesoría de estos expertos"1.

\section{La eficiencia en el logro de los resultados}

La evaluación de eficiencia se relaciona principalmente con el análisis económico del programa en términos de los costos y la factibilidad económica de la estrategia escogida. Los resultados se presentarán a través de índices como son el costo promedio de producir cada uno de los materiales, el costo de dotación de una escuela de todo el paquete de materiales y, finalmente, el costo que representaría dotar a todas las escuelas del material elaborado.

\section{CUADRO 7}

\begin{tabular}{|c|c|c|c|c|c|c|c|c|}
\hline $\begin{array}{l}\text { Área en el cual se } \\
\text { Desarrolló el seminario }\end{array}$ & $\begin{array}{l}\text { Año } \\
1969\end{array}$ & $\begin{array}{l}\text { Año } \\
1970\end{array}$ & $\begin{array}{l}\text { Año } \\
1971\end{array}$ & $\begin{array}{l}\text { Año } \\
1972\end{array}$ & $\begin{array}{l}\text { Año } \\
1973\end{array}$ & $\begin{array}{l}\text { Año } \\
1974\end{array}$ & $\begin{array}{l}\text { Año } \\
1975\end{array}$ & Totales \\
\hline Guía para el maestro de lo. & 18 & 20 & 2 & 119 & 372 & 127 & 112 & 770 \\
\hline Guía para el maestro de 20. & & 1 & 19 & 120 & 234 & 238 & 256 & 868 \\
\hline Guía para el maestro de 30. & & & & 7 & 217 & 276 & 268 & 768 \\
\hline Guía para el maestro de 40. & & & & & & 261 & 156 & 417 \\
\hline Guía para el maestro de 50 . & & & & & & & 126 & 126 \\
\hline Obras manuales y dibujo & 3 & 12 & 15 & 26 & 94 & 164 & 172 & 486 \\
\hline Música & & 2 & 4 & 26 & 65 & 118 & 122 & 337 \\
\hline Educación física & 9 & 20 & 29 & 33 & 76 & 192 & 183 & 542 \\
\hline Totales & 30 & 55 & 69 & 331 & 1.058 & 1.376 & 1.395 & 4.314 \\
\hline
\end{tabular}

El total de la producción de materiales durante la vigencia del proyecto ascendió a 171 millones de pesos, de los cuales el $61 \%$ se realizó con financiación colombiana y el resto con aportes nacionales.

\section{a) Costo unitario de los materiales}

El Cuadro 8 presenta un resumen de los costos de producción de los materiales en el año de 1974; las guías para el maestro oscilan entre $\$ 20$ y $\$ 170.00$; los textos para alumnos fluctúan entre $\$ 4.33$ y $\$ 7.50$, siendo notorio el bajo costo de la cartilla "Vamos a Leer" cuyo costo unitario fue de $\$ 1.44$. De los materiales didácticos los de mayor costo eran el fichero y los sellos, tal vez por la madera de los empaques. Al analizar la información a través de los años el incremento en los costos de los materiales concuerda con el comportamiento general de los precios en el país en su conjunto, excepto para la guía Calculemos, cuyo costo unitario pasa de $\$ 3.32$ en un año a $\$ 14.63$ en otro.

Si se tiene en cuenta lo establecido en la Resolución 2289 de 1975 en la cual se fijaba a los maestros un precio de $\$ 20, \$ 30, \$ 40$, y $\$ 50.00$ por las guías de lo. y 2o., 3o., 4o. y

\footnotetext{
${ }^{1}$ Nota canjeada entre el Embajador de la República Federal de Alemania y el Ministro de Relaciones Exteriores de Colombia.
} 
5o. Respectivamente, vemos que el subsidio estatal sería casi nulo para los primeros grados, del $50 \%$ para los de 40 . grado y del 30\%para la del 5o. grado. La filosofía de acuerdo con esta resolución era establecer un Fondo Rotatorio para publicar y divulgar guías adicionales. Sin embargo, al parecer y según lo explicado anteriormente esta estrategia no funcionó por falta de controles. Como punto de comparación en cuadro aparecen los costos a 1981 de los materiales elaborados por el ICCE, los cuales son prácticamente los mismos diseñados por la Misión Pedagógica Alemana, siendo el fichero el de mayor erogación, representando un $36 \%$ del costo total de los materiales.

\section{b) Costo anual de dotación de una escuela}

Para efecto de conocer los costos en los que se incurren para la dotación de una escuela, CDE, se ha asumido un modelo para dicha estimación con dos variantes o alternativas, aplicable a diferentes circunstancias, de las cuales se habla un poco más adelante.

En principio, se establece la definición del costo de dotación, en términos de los Agregados conformados por los elementos utilizados por el docente, el educando y el aula. Es decir, se entiende que dicho costo lo conforma la suma de los costos de los libros producidos con destino a la utilización por parte del maestro; la suma de los textos para el alumno y la suma de los costos de los elementos que constituyen el conjunto del material didáctico.

$$
\underset{P}{\mathrm{CDE}=} \underset{\mathrm{A}}{\Sigma \mathrm{CL}}+\underset{\mathrm{CL}}{(\mathrm{N})+\Sigma \mathrm{CMD}}
$$

De este modo, integran cada uno de los componentes del costo los siguientes elementos:

a) $\Sigma C L$ (Costos libros para el profesor):

$P$

- guías para el maestro (5 tomos)

- guías Calculemos (5 tomos)

- textos de música (2 tornos)

- textos de manualidades (2 tomos)

- textos de educación física y deporte (3 tomos)

--- textos de geografía general

b) $\Sigma \mathrm{CL}$ (Costo libros para estudiante):

A

- textos Calculemos (5 tomos)

- texto Vamos a Leer

- $\mathrm{N}$ : lo constituye el número de alumnos

c) $\Sigma$ CMD (Costos del material didáctico):

--- Fichero

- lotelect

- sellos de matemáticas, geografía

- fracciograf 
- cuerpos geométricos

- metros y decímetros cúbicos

- mapas

- láminas de historia y biología

- discos para enseñanza de música

- seguetas y bloques lógicos

La Alternativa 1 consiste en determinar los costos de dotación de una escuela y de cada alumno de educación primaria, bajo el supuesto de que se dotaría una escuela de cinco grados y doscientos estudiantes, con un solo conjunto de elementos producidos por la Misión, con la natural aclaración de que para el caso de los alumnos se les proporcionaría los textos según el número que hubiese en cada uno de los grados. Es decir, los estudiantes de primero recibirían cada uno el Calculemos 1 y el Vamos a Leer, que son los textos asignados para este grado; los del segundo recibirían el Calculemos 2; los de tercero el Calculemos 3, y así sucesivamente.

$$
\mathrm{CDE}=\underset{P}{\Sigma \mathrm{CL}}+\sum \mathrm{CL}(\mathrm{N})+\Sigma \mathrm{CMD}
$$

De este modo está dada por la segunda variable del modelo, la cual se redefine así:

$n=5$
$\sum$
$n^{\prime}=1$

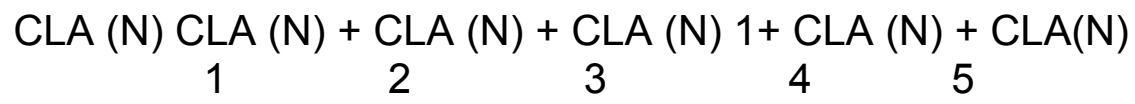

En donde:

$\mathrm{CLA}_{1}$ costo libros alumnos de primer grado.

$\mathrm{CLA}_{2}$ costo libros alumnos segundo grado.

$\mathrm{CLA}_{3}$ costo libros alumnos tercer grado.

$\mathrm{CLA}_{4}=$ costo libros alumnos cuarto grado

$\mathrm{CLA}_{5}=$ costo libros alumnos quinto grado .

$\mathrm{N}=$ número de alumnos para cada grado, que en este caso se establece en cuarenta estudiantes como número óptimo para cada grado de educación primaria.

Con el supuesto anotado, referente al hecho de que cada escuela utilizaría un solo conjunto de materiales, no se hablaría en estas dos alternativas de costos de dotación de un aula, sino del establecimiento. Pero sí se puede establecer el costo promedio de dotación por alumno (CPDA).

$$
\mathrm{CPDA}=\frac{\mathrm{CDE}}{\mathrm{N}}
$$

En donde el costo promedio de dotación del establecimiento se relaciona con $\mathrm{N}$, que es el número total de estudiantes en una escuela, el cual se determina en 200 alumnos. 
Definida de este modo la operacionalidad del modelo y utilizando los cálculos mostrados en los cuadros tenemos:

$\mathrm{CPDE}=\mathrm{CLP}+\mathrm{CLA}(\mathrm{N})+\mathrm{CMD}$

$C L P=666.64$

$$
\begin{aligned}
& C L A=230.80+168.80+196.40+214+300 \\
& C L A=1.110 \\
& C M D=4.789 .67
\end{aligned}
$$

CPDE $=666.64 \pm 1.110+4.789 .67=6566.31$

Costo promedio de Dotación por Alumno para la Alternativa 1

(Promedio de 1974).

$$
\mathrm{CPDA}=\frac{\mathrm{DPDE}}{\mathrm{N}} \quad \frac{6.566 .31}{200}=32.83
$$

Alternativa 2. Aproximando el análisis a un mayor nivel de exactitud en cuanto al tratamiento de los agregados con los cuales se vienen trabajando. Se plantea la necesidad de considerar una nueva posibilidad analítica. Ella tiene que ver con un criterio mucho más funcional en la utilización de los materiales por parte de los maestros, los alumnos y las aulas mismas, por cuanto los supuestos de dotación responden más a los requerimientos de cada grado de la educación primaria.

Es así como la redefinición del modelo para la alternativa 2 pasa por ubicar claramente los textos, guías y materiales didácticos que son destinados para cada uno de los cursos de primaria.

Con esta precisión, el modelo es aplicado para el caso de un establecimiento de cinco grados y cuarenta alumnos por grado, es decir, doscientos estudiantes por escuela.

De este modo, la diferencia con las alternativas anteriores estriba en que un establecimiento no utiliza un solo conjunto de materiales, sino que cada curso, cada profesor y cada estudiante dispondrán de los materiales, textos y guías que corresponden al grado para el que fueron producidos.

Con ello, la mencionada redefinición del modelo es:

$$
\mathrm{CDE}=\sum_{n=1}^{\mathrm{n}=5} \mathrm{CLP}+\sum_{\mathrm{n}=1}^{\mathrm{n}=5} \mathrm{CLA}(\mathrm{N})+\sum_{n=1}^{\mathrm{n}=5} \mathrm{CMD}
$$

En donde:

$\mathrm{n}=5$

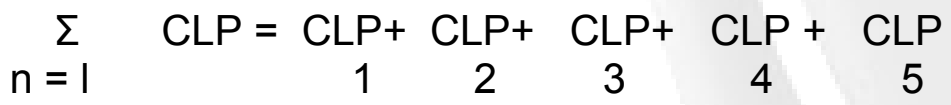


es decir, CLP 1 es el costo de todos los textos y guías del profesor del primer grado; CLP $_{2}$, el costo de los del profesor de segundo; $\mathrm{CLP}_{3}$, el costo de los del profesor de tercero; etc.;

$$
\sum_{n=1}^{\mathrm{n}=5} \operatorname{CLAn}(\mathrm{N})=\mathrm{CLA}_{1}(\mathrm{~N})+\mathrm{CLA}_{2}(\mathrm{~N})+\mathrm{CLA}_{3}(\mathrm{~N})+\mathrm{CLA}_{4}(\mathrm{~N})+\mathrm{CLA}_{5}(\mathrm{~N})
$$

tal como se definió,

$$
\underset{n=1}{\mathrm{n}=5} \mathrm{CMDn}=\mathrm{CMD}_{1}+\mathrm{CMD}_{2}+\mathrm{CMD}_{3}+\mathrm{CMD}+\mathrm{CMD}_{5}
$$

$\mathrm{CMD}_{1}$ es el costo del material didáctico utilizado exclusivamente en el primer grado, $\mathrm{CMD}_{2}$ en el costo del material didáctico del segundo; $\mathrm{CMD}_{3}$ en el costo del material del tercero, etc.

Alternativa 2 (promedio 1974).

$$
\begin{aligned}
& \text { CLP }=186.69+195.81+243.33+310.57+388.04 \\
& \begin{aligned}
C L P & =1.323 .44 \\
C L A & =5.77(40)+4.22(40)+4.91(40)+5.35(40)+7.50(40)
\end{aligned} \\
& \begin{aligned}
C L A & =1.110 \\
& =2.940 .15+2.756 .46+2.373 .74+2.373 .74+2.373 .74
\end{aligned} \\
& \begin{aligned}
C N D & =12.817 .83
\end{aligned} \\
& \text { CPDE }=1.324 .44+1.110+12.817 .83=15.252 .27
\end{aligned}
$$

En lo que respecta a los costos promedio por cursos (CPCn), se plantea que para el primer grado es:

$$
\mathrm{CPC}_{1}=\mathrm{CPLP}_{1}+\mathrm{CPLA}_{1}(\mathrm{~N})+\mathrm{CPMD}_{1}
$$

y así sucesivamente para los cuatro grados restantes, en donde:

$\mathrm{CPLP}_{1}=$ costo promedio libros para profesores de primero, $\mathrm{CPL} \mathrm{A}_{1}=$ costo promedio libros para alumnos de primero.

$\mathrm{CPMD}_{1}=$ costo promedio material didáctico para primero y $\mathrm{N}=$ número de estudiantes de primer grado (40 alumnos).

Con una definición semejante se calculan los costos promedios para los cursos segundo, tercero, cuarto y quinto, utilizando un número de alumnos $(\mathrm{N})$ igual a 40; número éste que como se dijo antes, se considera como óptimo para el funcionamiento de un grado normal en primaria.

De este modo tenemos:

Costo promedio por curso para alternativa 2 (Promedio de 1971). 
A. Para el primer curso:

$$
\begin{aligned}
& \mathrm{CPC}_{1}=186.69+5.77(40)+2.940 .15 \\
& \mathrm{CPC}_{1}=3.357 .64
\end{aligned}
$$

B. Para el segundo curso:

$$
\begin{aligned}
& =195.81+4.22(40)+2.756 .46 \\
& =3.121 .07
\end{aligned}
$$

C. Para el tercer curso:

$$
\begin{aligned}
& =243.33+4.91(40)+2.373 .74 \\
& =2.813 .47
\end{aligned}
$$

D. Para el cuarto curso:

$$
\begin{aligned}
& =310.57+5.35(40)+2.373 .74 \\
& =2.898 .31
\end{aligned}
$$

E. Para el quinto curso:

$$
\begin{aligned}
& =388.04+7.50(40)+2.373 .74 \\
& =3.061 .78
\end{aligned}
$$

Costo promedio de alumno por curso para Alternativa 2.

A. Para alumnos del primer curso:

$$
\mathrm{CPAC}_{1}=\frac{\mathrm{CPC}_{1}}{\mathrm{~N}}=\frac{3.357 .64}{40}=83.94
$$

B. Para alumnos del segundo curso:

$$
\mathrm{CPAC}_{2}=\frac{\mathrm{CPC}_{2}}{\mathrm{~N}} \quad \frac{3.121 .07}{40}=78.03
$$

C. Para alumnos del tercer curso:

$$
\mathrm{CPAC}_{3}=\frac{\mathrm{CPC}_{3}}{\mathrm{~N}} \quad \frac{2.813 .47}{40}=70.34
$$

D. Para alumnos del cuarto curso:

$$
\mathrm{CPAC}_{4}=\quad \frac{\mathrm{CPC}_{4}}{\mathrm{~N}} \quad \frac{2.898 .31}{40}=72.46
$$


E. Para alumnos del quinto curso:

$$
\mathrm{CPAC}_{5}=\frac{\mathrm{CPC}_{4}}{\mathrm{~N}} \quad \frac{3.061 .78}{40}=76.54
$$

\section{c) Costos de dotación para 1981}

Con el objeto de conocer cuánto le costaría al país en 1981 la dotación de una escuela de todos los materiales de la Misión Pedagógica Alemana, se trabajó con los índices de costo de vida. Pero sin duda la utilización de dicho indicador, tomando el total nacional, contaría con la dificultad de asumir el comportamiento del índice general como idéntico a las modificaciones en los precios de los elementos educativos. Como es sabido, en Colombia el índice de precios al consumidor se estima tomando en forma ponderada las variaciones de cuatro grupos, a saber: alimentos, vivienda, vestuario y misceláneo. En lo que al sector educativo se refiere, importa para cualquier tipo de inferencia sobre costos educativos, el último de estos grupos y, siendo más rigurosos para el caso que se viene tratando aquí, lo que hace referencia a los Artículos Escolares.

La validez de esta apreciación metodológica es aún mucho más importante si se tienen en cuenta los factores de ponderación en la construcción del mencionado índice general. En el grupo misceláneo en su conjunto, dicho factor para los artículos escolares es de 0.59, en lo que se refiere al índice de precios para obreros, que es con el cual se realizarán los cálculos a que se refieren los cuadros que más adelante se muestran.

En cuanto a los elementos que componen el subgrupo Artículos escolares, habría que mencionar las modificaciones que ellos sufren, al implementarse el cambio en la metodología del cálculo de los índices de precio por parte del DANE, a partir de 1978*. Con la exclusión de elementos como block, tinta y estilógrafos, se agrega a la lista de este subgrupo un artículo con el que antes no se contaba, como es el de textos de matemáticas para primaria y bachillerato. Aunque dos artículos más son incorporados (bolígrafos y revistas), es importante reconocer que el contar por lo menos con estos textos para la determinación de dicho índice, valida su utilización en este trabajo.

Los indicadores, permitieron concluir que los precios en noviembre de 1981, aumentaron en un $277.73 \%$ con respecto a 1974 . En razón a que en esta parte interesa conocer el esfuerzo financiero que tendría que asumir el Estado colombiano para la dotación de una escuela de los materiales de la misión colombo-alemana, se aplica este porcentaje de incremento a los resultados obtenidos según el desarrollo del modelo básico de este estudio. De igual modo, se plantean las dos alternativas centrales estudiadas en este análisis, en relación con los costos de estas posibilidades de dotación.

De este modo, si la tendencia en los aumentos de los precios en los Artículos Escolares, se asimila a la tendencia que da como resultado los precios que se pagarían en 1981 por la producción de los materiales de la Misión Pedagógica Alemana; los costos serían los que se muestran a continuación. También se registran en el siguiente cuadro los costos de libros para el profesor, para el alumno y para el material didáctico. Se recogen en este y los cuadros siguientes los cálculos resultantes del modelo para 1974, con el fin de visualizar las diferencias entre un costo y otro, en los años tomados como puntos de referencia (Ver Cuadro 9).

\footnotetext{
"Indice de precios de Art ículos Escolares para Bogotá en diciembre. 
Aceptado el supuesto de la asimilación de las tendencias en los precios con la del costo promedio de dotación de una escuela, se diría que para la alternativa 1 , o sea la de que una escuela emplearía un solo conjunto de materiales sería en noviembre de 1981 de \$ 24.802.00 aproximadamente. Para la Alternativa 2, es decir, que cada maestro, alumno y aula utilizaría los elementos que correspondan al grado escolar respectivo, ascendería a \$ 57.612.40 aproximadamente.

\section{CUADRO 9}

\begin{tabular}{|lccrr|}
\hline \multicolumn{4}{|c|}{$\begin{array}{c}\text { COSTO PROMEDIO DE DOTACION POR ESCUELA Y SUS AGREGADOS } \\
\text { EN LAS ALTERNATIVAS 1 Y 2 PARA }\end{array}$} \\
& \multicolumn{4}{c}{ 1974 Y 1981 } \\
\hline \multicolumn{4}{c}{ Año 1974 } & \multicolumn{2}{c|}{ Año 1981 } \\
& Alternativa 1 & Alternativa 2 & Alternativa 1 & Alternativa 2 \\
\hline CPL & 666.64 & 1.324 .44 & 2.518 .09 & 5.002 .80 \\
OLA (N) & 1.110 .00 & 1.110 .00 & 4.192 .80 & 4.192 .80 \\
CMD & 4.789 .67 & 12.817 .83 & 18.092 .02 & 48.416 .79 \\
CPDE & 6.566 .31 & 15.252 .27 & 24.802 .91 & 57.612 .39 \\
\hline
\end{tabular}

Relacionando estos costos con las estadísticas siguientes, se puede conocer de modo aproximado también, lo que costaría al país el cubrimiento de materiales para todas las escuelas de primaria que funcionan en Colombia. En el Cuadro 10, se muestra la última información oficial suministrada por el Ministerio de Educación Nacional.

Si con esta información se supiese que todas las 35.118 escuelas contasen con cinco grados y que cada grado tuviese 40 estudiantes (cuestión bastante improbable, pero fue imposible lograr una estadística más desagregada para 1980), se encontraría que para la alternativa 1 el costo total sería de más de 870 millones de pesos y para la Alternativa 2 , ascendería a más de 2 mil millones de pesos.

Contando con la restricción que presenta la estadística del Cuadro 10, habría que anotar también que los precios de los textos escolares en Colombia, se hallan bajo control oficial. Estas limitantes del análisis, no lo invalidan en su totalidad, por cuanto que el propósito teórico se cumple, al aportar a través de él, un conjunto de elementos de juicio de la mayor importancia, para los posteriores desarrollos en materia de producción y distribución de textos, materiales y ayudas educativas.

Con esta intención, se muestran a continuación los costos promedio por curso (CPC) y los costos promedio por alumno (CPA), derivados de los cálculos iniciales para 1981, contemplados también para las dos alternativas analizadas y empleando el mismo procedimiento técnico del anterior cálculo (Ver Cuadro 11).

De igual modo, la utilidad de presentar los costos por curso y alumno en la Alternativa 2, cuenta con la importancia de destacar un esfuerzo financiero desagregado para cada uno de los grados que componen la educación primaria. De acuerdo con los relativos altos costos señalados aquí y con las ventajas pedagógicas que proporciona la utilización de los materiales de la Misión Pedagógica Alemana — de lo cual se habla en otra parte de este estudio - se deriva la importancia de contar con estos instrumentos cualitativos para 
las posteriores realizaciones de un proyecto de la naturaleza del que se ocupa esta evaluación (Ver Cuadro 12).

Quizá un cálculo un poco más realista tendiente a estimar el costo de dotación para el año 81 , sería relacionar el costo promedio por alumno con el número total de alumnos. Para el caso de esta Alternativa 1 , el monto del esfuerzo financiero ascendería a más de 517 millones de pesos.

CUADRO 10

\begin{tabular}{|c|c|c|c|}
\hline \multicolumn{4}{|c|}{$\begin{array}{c}\text { EDUCACION PRIMARIA } \\
\text { PERSONAL DOCENTE, MATRICULA Y ESTABLECIMIENTOS } \\
\text { SEGUN SECTOR } 1975-1980\end{array}$} \\
\hline & & 1975 & 1980 \\
\hline 1. & Personal docente & & \\
\hline & Oficial & 98.039 & 112.851 \\
\hline & Privado & 23.918 & 26.370 \\
\hline & Total & 121.957 & 139.221 \\
\hline 2. & Número de alumnos & & \\
\hline & Oficial & 3.318 .577 & 3.548 .166 \\
\hline & Privado & 592.067 & 625.068 \\
\hline & Total & 3.911 .244 & 4.173 .234 \\
\hline 3. & Número de Escuelas & & \\
\hline & Oficial & 28.666 & 30.498 \\
\hline & Privado & 4.536 & 4.620 \\
\hline & Total & 33.202 & 35.118 \\
\hline
\end{tabular}

Fuente: "Estadísticas Educativas 1966 - 1980". MEN, Oficina de Planeación Educativa, Bogotá, 1981. (Mimeógrafo).

CUADRO 11

\begin{tabular}{|lcc|}
\hline & \multicolumn{2}{c|}{$\begin{array}{c}\text { COSTO PROMEDIO POR CURSO Y } \\
\text { POR ALUMNO (ALTERNATIVA 1) }\end{array}$} \\
\hline & 1974 & 1981 \\
\hline CPC & 1.313 .20 & 4.960 .35 \\
\hline CPA & 32.83 & 124.01 \\
\hline
\end{tabular}

\section{d) La utilización de los materiales}

La efectividad en el logro de los objetivos de la Misión se mide en último término por la utilización que los maestros hayan hecho de los materiales y por la permanencia de su 
uso cinco años después de terminado el proyecto. Como indicadores de efectividad se tomaron la presencia de los materiales en los establecimientos públicos de educación primaria, su utilización por parte del maestro, la forma como los están usando y la percepción de la utilidad que le han prestado en su labor docente.

Para desarrollar este aspecto de la evaluación se realizó una visita a los planteles educativos y se levantó un inventario de la existencia de materiales y su estado de conservación. Además se aplicó un cuestionario a maestros a través del cual se recogía información sobre la posesión de los materiales, la forma como se adquirieron, la ayuda prestada por cada uno de ellos y la capacitación que se recibió para su manejo.

\section{CUADRO 12}

\begin{tabular}{|lcccc|}
\hline \multicolumn{5}{|c|}{$\begin{array}{l}\text { COSTO PROMEDIO POR CURSO Y POR ALUMNO } \\
\text { (ALTERNATIVA 2) }\end{array}$} \\
\hline Curso & CPC & CPA & CPG & CPA \\
\hline Primero & 3.357 .64 & 83.94 & 1981 & \\
Segundo & 3.121 .07 & 78.03 & 11.789 .21 & 294.74 \\
Tercero & 2.813 .47 & 70.34 & 10.627 .32 & 265.69 \\
Cuarto & 2.898 .31 & 72.46 & 10.947 .78 & 273.70 \\
Quinto & 3.061 .78 & 76.54 & 11.565 .26 & 289.11 \\
\hline
\end{tabular}

Para la escogencia de la muestra se utilizó un diseño polietápico con selección controlada, que asegura una adecuada representación tanto de las diversas regiones y zonas del país como de las distintas modalidades de escuelas primarias. Además como se trabajó bajo restricciones de tiempo y presupuesto, se buscó agilizar y minimizar los costos de recolección de la información.

Para la determinación del tamaño de la muestra se escogió un nivel de significación del .05 y una precisión del $5.6 \%$ quedando un total de 303 maestros para encuestar, tomando como variable a estimar la proporción de maestros que utilizan los materiales. Este raciocinio permite utilizar la presunción de máxima varianza en la fórmula:

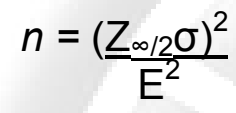

Donde,

$n=$ Tamaño de la muestra

$Z_{\infty / 2}=\quad$ Nivel de confiabilidad

$\sigma=$ Desviación típica de la variable a estimar

$\mathrm{E}=$ Precisión de la estimación

La varianza de una variable dicotómica medida como proporción llega a su máximo cuando el porcentaje de casos favorables, los que utilizan los materiales, es el 50 \%. Por lo tanto, al aplicar la fórmula anterior se tiene:

$$
\left(\underline{1,96)^{2}} \cdot 5^{2}=303\right.
$$


Con base en un primer sondeo se seleccionaron cuatro departamentos localizados en las cuatros grandes regiones del país:

Bolívar, región norte

Antioquia, región Nororiental

Boyacá, región Central

Valle, región Sur oriental

El Departamento del Valle tuvo que ser eliminado porque en el momento de la encuesta se encontraba en período de vacaciones. Finalmente se eligieron los departamentos de Bolívar, Antioquia y Boyacá.

Con base en los listados de escuelas receptoras se asignó un número de $70 \%$ de maestros en el área urbana y $30 \%$ en el área rural por departamento, suponiendo que por escuela urbana se entrevistarían cinco (5) maestros y por rural dos (2) maestros.

Se visitaron un total de 99 establecimientos en los departamentos de Antioquia, Bolívar y Boyacá y se aplicó el cuestionario a 310 maestros.

En la parte A del cuestionario se recogió información sobre todos los maestros de la escuela para determinar la proporción de docentes que habían empleado los materiales. La parte $B$ se aplicó sólo a quienes los habían utilizado, escogiendo al azar un maestro por cada uno de los cursos existentes en el plantel.

En cuanto a la existencia de los materiales en las escuelas se encontró que las "guías" eran las de mayor disponibilidad, mientras que otros textos como el de Geografía y Trabajos Manuales sólo se encontraron en un 5\% de los establecimientos (Cuadro A-6). Tal vez esta poca accesibilidad se deba a la calidad de la impresión ya que éstos se caracterizaron por ser los más lujosos. Los materiales didácticos se encontraban en casi la mitad de las escuelas, lo cual es una cifra cercana a su cubrimiento potencial. Fue notorio el hecho de que había mayor proporción de escuelas rurales que contaban con el material, siendo tal vez mayor la valoración que de éstos se hace en esta área debido a su lejanía del mercado de otros textos o materiales.

Al desagregar la disponibilidad del material por el estado o calidad actual del mismo se encuentra que aproximadamente el $75 \%$ está en buen o excelente estado. Tal vez la excepción la constituye el fichero, donde casi la mitad se encuentra en precarias condiciones. El gran mueble de madera es utilizado desde soporte para las plantas hasta - con una pequeña modificación que consiste en quitar una tabla de por medio- en sitio para guardar el calzado en los dormitorios de las niñas internas de una anexa. Afortunadamente los nuevos ficheros que está distribuyendo el ICCE se han hecho más funcionales que el diseño inicial.

Al medir cuál ha sido el grado de utilización de las "guías", es sorprendente el alto porcentaje de maestros que declararon haberlas utilizado alguna vez, el $96 \%$, lo cual hace asumir que las "guías" se han constituido en punto de referencia obligatorio para el maestro. 
Adicionalmente, un $7 \%$ de los maestros entrevistados dijeron poseer las "guías" en ese momento. Una cuarta parte de los maestros dijo haber obtenido las guías comprándolas al Ministerio, alcanzando esta proporción un $65 \%$ si se agregan los que están usando la del establecimiento. El porcentaje de los que la compraron en librería es bajo, el 3\%, constatando la existencia de canales diferentes al Ministerio para su adquisición.

Al indagar sobre el material empleado para preparar y dictar la clase se encontró como el medio de mayor utilización el "cuaderno del profesor". Para el total de maestros, un 40\% utiliza el cuaderno, el cual es tomado de las guías en el $10 \%$ de los casos, del 1710 en el $4 \%$, y de las guías y el 1710 en el $26 \%$ de los casos. En el área rural el uso del "cuaderno del profesor es mayor, $55 \%$ de los casos, para cuya elaboración el $50 \%$ usó como fuente las guías y el 1710. Este medio es muy generalizado entre los docentes de primaria y en muchas ocasiones es el libro de apuntes de la normal o de maestros antiguos el instrumento usado para "transmitir" los conocimientos a los alumnos. Las guías como único medio de dictar clase fue encontrado en el $5 \%$ de los casos y en forma complementaria en el $62 \%$, sea como texto o como base para elaborar el cuaderno.

Al preguntársele al maestro sobre la ayuda que le prestan las guías para el desarrollo de las diversas asignaturas, se obtuvo la siguiente información: para las áreas de física y química cerca del $30 \%$ de los maestros estuvieron de acuerdo en que eran una buena ayuda pero de difícil utilización. Estas respuestas difieren de las demás áreas como son ciencias naturales, lenguaje, trabajo manual y educación física donde la calificación como "buena ayuda" llega al $80 \%$. La dificultad en las áreas de física y química tal vez es explicable por su intensificación ya que anteriormente eran tratadas en forma muy general, como se vio en el capítulo sobre desarrollo curricular.

Otro punto importante dentro de la evaluación de las guías era la valoración por parte del maestro de los contenidos y la metodología utilizada.

Casi una cuarta parte de los maestros respondió afirmativamente ante la pregunta de si tuvieron dificultad en la utilización de las guías. La proporción de maestros a quienes se les dificulta su uso parece aumentar a medida que avanza la primaría, así la proporción de los maestros en el curso 4o. es del $32 \%$, mientras que en los de 10 . Es del $17.5 \%$.

Si se compara el grado de dificultad experimentado con el nivel académico alcanzado por el maestro, se observa que es mayor a medida que el nivel baja, pero también se da en los docentes que tienen educación universitaria incompleta. Para los docentes con formación pedagógica, los normalistas, la proporción es menor.

Al preguntársele a los maestros la razón por la cual tuvieron dificultad, la gran mayoría cree $(43 \%)$ que faltó capacitación para su utilización. La falta de claridad fue otra de las razones mencionadas (16\%), así como la dificultad para manejarlas (11\%).

La capacitación de los maestros formaba parte integral de la estrategia del suministro de guías, suponiéndose que serían entregadas al finalizar los cursos de entrenamiento. Sin embargo, vemos que esta estrategia no funcionó del todo. Primero por su cobertura: un $43 \%$ de los maestros declararon no haber asistido a ningún curso. De otra parte aquellos que sí, hicieron un entrenamiento muy corto: las tres cuartas partes de los maestros del área rural dijeron haber participado en cursos de una semana o menos. En opinión del $70 \%$ la capacitación recibida no fue suficiente. 
Desde esta perspectiva se puede decir que la implementación de los puntos centrales de la estrategia planteada por la Misión Pedagógica Alemana, la distribución del material y la capacitación de los docentes, se quedó corta frente a las metas de producción de materiales.

Finalmente, quiso obtenerse información sobre el aspecto físico de las guías, siendo la opinión de la mayoría de los maestros que tanto el tamaño como el tipo de letra, clase de papel y dibujos habían sido acertados, la excepción la constituyó el tipo de encuadernación que fue considerada de mala calidad, ya que las guías se desempastan muy fácilmente. Un $16 \%$ también sugirió que las guías deberían tener un mayor número de ilustraciones. Contrariamente a lo que sucedió con las guías, el uso de los demás textos para profesores fue bastante bajo, no llegando la proporción de maestros que dijeron haberlos empleado alguna vez al 25\%. Este bajo índice puede atribuirse principalmente a problemas de distribución y pérdida del material, ya que este material se imprimió en número suficiente para que cada maestro contara al menos con un ejemplar.

Es importante también llamar la atención sobre el aparente fracaso de la estrategia de suministro de libros a los estudiantes, cuya utilización no superó para ningún departamento el $20 \%$. Las causas son múltiples. Unas se relacionan con la falta de un apoyo por parte del nivel decisorio del Ministerio al suministro de textos subsidiados o gratuitos a los alumnos de la escuela primaria. Esto conllevó a una ineficiencia en los canales de distribución, de una parte, y a una prematura paralización de la impresión de otros materiales cuando estaban en artes finales. Sin embargo, los alumnos siguen adquiriendo los textos de casas editoriales particulares, significando un crecimiento en los costos educativos en las clases menos favorecidas.

Dentro de la operatividad y la implementación de esta estrategia también se presentaron fallas, como es la falta de previsión para el reemplazo de aquellos ejemplares de difícil utilización, lo cual desembocó por parte de los maestros en el abandono de la utilización de los textos para alumnos al no encontrarse el número de ejemplares completos.

Al incluirse en el formulario dos preguntas referentes a este tema, se encontró que cerca del $80 \%$ de los maestros que contestaron califican a los libros como buenos o excelentes, tanto en su metodología como contenidos.

Así mismo se les preguntó su opinión sobre el suministro de textos a los alumnos, sea como donación o subsidio. Cerca del $70 \%$ de los encuestados se mostraron de acuerdo con la política del suministro gratuito; en caso de no adoptarse esta solución, un $45 \%$ estuvo de acuerdo en que el Estado debería vender baratos los textos y sólo un $2 \%$ cree que los alumnos deben comprar los textos.

De todas maneras es importante que se haga un análisis más detallado de esta estrategia tanto desde el punto de vista operativo como cualitativo, ya que como se verá más adelante el uso de textos diferentes a los oficiales es bastante generalizado dentro del magisterio.

Se hizo también un sondeo sobre la utilización de las distintas ayudas educativas. Entre estas hay algunas de uso generalizado como son las figuras geométricas que han sido utilizadas por el $43 \%$, el globo terrestre por un $40 \%$, los sellos de matemáticas por un $36 \%$, o los mapas o las láminas de biología, por más de un $30 \%$. De otra parte, al comparar el uso actual del material por los maestros del curso al cual va dirigido, vemos, por ejemplo, que 47 maestros de 62 que hay en el curso primero, están usando el fichero. 
El uso de las figuras geométricas y de las láminas de biología también es bastante común según estos porcentajes. El juego de arco y segueta es el menos utilizado junto con los frisos de historia. Recordemos que el primero de estos existe sólo en el $2 \%$ de los planteles visitados. Así mismo este juego fue calificado de inútil por parte de más de las tres cuartas partes de los maestros, siendo el resto de materiales considerados de mucha utilidad.

Al pedírsele a los maestros que calificaran la ayuda que le prestaron los materiales de la Misión, más de un $90 \%$ estuvo de acuerdo en que fueron una gran ayuda para la introducción de temas nuevos y como medio para mejorar el aprendizaje. El 15\% respondió que eran deficientes como medio para mejorar la metodología de la enseñanza.

En general puede observarse que los maestros tienen una actitud positiva hacia todo el conjunto de materiales, 118 maestros creen que las guías deberían volverse a producir, 44 opinan que esto debe hacerse para todo el material. Como regla general se encontró que los materiales de la MPA son los únicos materiales existentes en las escuelas. Otras ayudas que ellos usan, mapas, láminas, son elaboradas en la propia escuela o adquiridas en empresas particulares. El $80 \%$ de los maestros respondió que posterior a los materiales de la Misión no han recibido otro por parte del Ministerio, excepto para los materiales de los programas experimentales, siendo el caso de 11 maestros.

Respecto a la utilización de otros textos, se encontró que casi un $70 \%$ de los maestros utiliza textos complementarios que adquieren en librerías. El número de títulos fue bastante alto para todas las asignaturas.

\section{Qué podemos aprender de esta experiencia}

Como puede deducirse de las páginas precedentes la labor realizada por la Misión Pedagógica Alemana no puede pasar inadvertida dentro de la historia educativa colombiana. En primer lugar por la magnitud de la labor emprendida: casi la totalidad de los maestros tanto del área urbana como rural, han utilizado los materiales producidos por la Misión. Para la mayoría de ellos estos materiales han sido el único punto de referencia y orientación acerca de los contenidos y la metodología que debe impartirse dentro de la escuela primaria. Tal vez por este motivo más de la mitad de los maestros creen que estos materiales deben reimprimirse y entregárseles de nuevo. Un segundo factor, se refiere a la amplitud de la tarea desarrollada y a la cantidad de esfuerzos humanos y financieros necesarios para su realización. Se trataba de desarrollar un "paquete integral" para el uso del maestro con los elementos que hicieran posible el mejoramiento del proceso de enseñanza-aprendizaje, así como la capacitación para su uso. Financieramente esta experiencia significó una erogación de 171 millones de pesos, 61\% a cargo del gobierno alemán y el resto del gobierno colombiano.

Operativamente se dieron a la tarea de desarrollar el material un grupo de aproximadamente 25 técnicos colombianos quienes junto con 6 expertos alemanes trabajaron durante 10 años, cuyo resultado es la producción de las guías de enseñanza para los cursos lo. a 5o. de primaria, otros textos para las áreas de geografía, música, trabajos manuales, y las ayudas educativas necesarias para las diversas asignaturas de los cursos. El material impreso tenía un tiraje suficiente para llegar a la totalidad de los maestros y alumnos que en ese momento se encontraban en el sistema educativo oficial del país. 
El proyecto fue muy innovativo en áreas como la música y la educación física, áreas todavía incipientes dentro del desarrollo programático colombiano. Se le puede también atribuir el pretender entregar al magisterio no sólo los contenidos de las diversas asignaturas sino también la metodología para su desarrollo, aspecto que superaba el tradicional programa del 1710 entregado a los maestros. Se hizo así mismo un primer intento de integrar las diversas asignaturas, al menos para los primeros años, con un enfoque de enseñanza globalizada. Se implementó un sistema de capacitación denominado "multiplicación de multiplicadores" que a pesar de presentar diversas fallas en su implementación tuvo una cobertura muy alta. Puede también atribuírsele a la Misión la puesta en marcha de un sistema de dotación de textos para los estudiantes de la escuela primaria, en las áreas de lectura y matemáticas.

Si el gobierno colombiano quisiera repetir la experiencia de proveer a todas las escuelas con el material producido por la Misión Pedagógica Alemana, la erogación oscilaría entre 870 y 2.000 millones de pesos ${ }^{\star}$, no siendo en ningún caso mayor de $\$ 300$ por alumno en un año. Al calculársele una vida útil al material de 5 años el costo alumno bajaría aun más. De instituirse, como fue la intención inicial, un sistema no de donación sino de subsidio a los maestros de un porcentaje del valor de los textos y materiales y con esto se conformara un sistema de fondo rotatorio la factibilidad de realización de un proyecto de esta naturaleza sería aún mayor.

Al comparar entonces los resultados presentados por el proyecto con las metas fijadas por los dos gobiernos en el convenio, hay que decir que se alcanzó una alta realización de las metas, sin embargo, se presentó una serie de experiencias que vale la pena subrayar a manera de conclusiones, a saber:

Organizativa mente la ubicación del proyecto le dio autonomía y agilidad administrativa para cumplir sus objetivos, convirtiéndose más tarde en un obstáculo para su continuidad en el Ministerio. La Misión fue considerada ajena a las dependencias que internamente tenían a su cargo el desarrollo curricular de la escuela primaria, hecho que desembocó en una prematura sustitución del material por el de la nueva reforma de 1976. Si bien esto no significó la pérdida del esfuerzo llevado a cabo por el grupo que trabajó bajo la asesoría alemana sí incidió en la efectividad con que hubiera podido terminarse el proyecto.

La implementación, especialmente en lo referente a la distribución y entrega del material careció de controles y mecanismos adecuados haciendo insuficiente la labor proyectada. Al parecer tampoco se tenían instrumentos de retro-alimentación que le permitieran al grupo central tomar las decisiones para corregir las anomalías en la distribución y uso del material.

En cuanto al material producido no todo tuvo el mismo impacto. De los textos impresos para el profesor las guías parecen ser las de mayor utilización, demeritándose así la efectividad de otros textos complementarios como fueron los elaborados en las áreas de geografía o trabajos manuales. No obstante su aparente fracaso se recomienda replantear la estrategia de distribución de textos para los alumnos, ya que como se demostró estos pueden ser elaborados a costos muy bajos, significando un gran alivio económico para los padres .

\footnotetext{
*Este cálculo fue hecho para 1981.

La diferencia la hace el sistema de dotación como puede verse en el texto correspondiente.

*Un estudio elaborado por Alba P. de Cárdenas demuestra cómo en algunas escuelas los requerimientos de elementos de uso individual puede constituir un $40 \%$ del costo total de la educación.
} 
La gran lección de lo que fue la experiencia de la Misión Pedagógica Alemana está en el campo del manejo de la política educativa, tanto a nivel nacional como internacional. Ya en 1972 se puntualiza sobre la necesidad de incorporar la asistencia técnica internacional a planes y programas educativos de largo plazo y acordes con las prioridades del sector, sin embargo en 1976 año en que se termino la reproducción de los materiales de la Misión el Gobierno Nacional se encontraba gestionando ante la AID un préstamo para la impresión de nuevos materiales educativos.

A nivel de la política educativa nacional el punto más importante es tal vez el de pretender introducir nuevas reformas al sistema sin que medie una evaluación de las experiencias previas, o lo que es más grave, sin darle el tiempo suficiente para que se muestren sus posibles beneficios.

\section{CUADRO A-1}

MATERIALES PRODUCIDOS POR LA MISION PEDAGOGICA ALEMANA

\begin{tabular}{|c|c|c|c|c|c|c|c|c|c|c|}
\hline Materiales & 1970 & 1971 & 1972 & 1973 & 1974 & 1975 & $\begin{array}{l}1975 \\
\text { Dic. }\end{array}$ & 1976 & 1978 & Totales \\
\hline Guías de 1er. Año & 10.000 & 10.000 & 20.000 & & & 20.000 & & $30.000^{* * *}$ & & 90.000 \\
\hline Guías de $2^{\circ}$. Año & & 10.000 & 20.000 & & & 20.000 & & $30.000^{* * *}$ & & 80.000 \\
\hline Guías de 3er. Año & & & & 10.000 & & 20.000 & & $20.000^{\star * *}$ & & 50.000 \\
\hline Guías de $4^{\circ}$. Año & & & & & 10.000 & 10.000 & & 10.000 & 10.000 & 40.000 \\
\hline Guías de $5^{\circ}$. año & & & & & & 10.000 & & 10.000 & 10.000 & 30.000 \\
\hline Calculemos 1 & 50.000 & 85.000 & $720.000^{*}$ & & 200.000 & 315.000 & & & & 1.370 \\
\hline Calculemos 2 & & 50.000 & $605.000^{\star *}$ & & 400.000 & & & & & 1.055 .00 \\
\hline Calculemos 3 & & & $470.000^{* *}$ & & 300.000 & & & & & 770.000 \\
\hline Calculemos 4 & & & & 285.000 & 100.000 & & & & & 385.000 \\
\hline Calculemos 5 & & & & & $250.000^{*}$ & & & & & 250.000 \\
\hline Guía Calculemos 1 & & 5.000 & 20.000 & 15.000 & $18.000^{*}$ & & & & & 58.000 \\
\hline Guía Calculemos 2 & & 5.000 & 10.000 & 10.000 & 16.000 & & & & & 41.000 \\
\hline Guía Calculemos 3 & & & 5.000 & 10.000 & 12.000 & & & & & 27.000 \\
\hline Guía Calculemos 4 & & & & & $8.000^{*}$ & & & & & 8.000 \\
\hline Guía Calculemos 5 & & & & & & 10.000 & & & & 10.000 \\
\hline
\end{tabular}

* Se hicieron dos ediciones en el año

** Se hicieron tres ediciones en el año

*** Total producido de 1976 a 1978

\section{CUADRO A-2}

\section{Materiales}

Vamos a leer 1

Cuadernos de instrucción

Viva la música I

Viva la música II

La clase alegre

Otra clase alegre

Educación física I

Educación física II

Juegos Nales. Escolares de

atletismo

Geografía general

\begin{tabular}{|c|c|c|c|c|c|c|c|c|c|}
\hline \multirow[t]{9}{*}{1970} & 1971 & 1972 & & & 1975 & $\begin{array}{l}1975 \\
\text { Dic }\end{array}$ & 1976 & 1978 & Totales \\
\hline & & & 1.200 .00 & $\begin{array}{r}1.200 .00 \\
30.000\end{array}$ & & & & & $\begin{array}{r}2.400 .00 \\
30.000\end{array}$ \\
\hline & 20.000 & 20.000 & 20.000 & 25.000 & & 20.000 & & 9.000 & 114.000 \\
\hline & & & 1000 & 20.000 & & & 10.000 & 9.000 & 39.000 \\
\hline & & & 10.000 & $\begin{array}{l}10.000 \\
10.000\end{array}$ & 10.000 & & $\begin{array}{l}10.000 \\
10.000\end{array}$ & $\begin{array}{l}9.000 \\
9.000\end{array}$ & $\begin{array}{l}39.000 \\
39.000\end{array}$ \\
\hline & & & 30.000 & 25.000 & & & 10.000 & 9.000 & 74.000 \\
\hline & & & 25.000 & 22.000 & & & 10.000 & 9.000 & 66.000 \\
\hline & 30.000 & & & & 5.000 & & & 5.000 & 40.000 \\
\hline & & & & & 10.000 & & & & 10.000 \\
\hline
\end{tabular}




\section{CUADRO A-3}

\section{MATERIALES PRODUCIDOS POR LA MISION PEDAGOGICA ALEMANA}

\begin{tabular}{|c|c|c|c|c|c|c|c|c|c|c|}
\hline Materiales & 1970 & 1971 & 1972 & 1973 & 1974 & 1975 & $\begin{array}{l}1975 \\
\text { Dic. }\end{array}$ & 1976 & 1978 & Totales \\
\hline Ficheros $^{\star \star \star \star}$ & 100 & & 1.050 & 1.000 & $2.000^{*}$ & 1.650 & 520 & 1.300 & $\begin{array}{c}1.995 \\
6.041^{* * *}\end{array}$ & 15.676 \\
\hline Lotelec & & & 1.000 & & $1.300^{*}$ & $2.000^{*}$ & 1.500 & 2.100 & $\begin{array}{c}2.000 \\
6.041^{* * *}\end{array}$ & 15.941 \\
\hline Sellolect & & & & & 179 & 2.600 & 2.000 & $2.000^{*}$ & $\begin{array}{c}2.000 \\
6.041^{* * *}\end{array}$ & 14.820 \\
\hline Sellomat & 500 & 1.050 & & 1.000 & 3.500 & & & 2.000 & $\begin{array}{c}2.000 \\
6.041^{* \star *}\end{array}$ & 16.091 \\
\hline Fracciograf & & & 1.000 & 2.000 & 1.000 & 1.000 & & 2.200 & $\begin{array}{c}2.000 \\
6.041^{* * *}\end{array}$ & 15.241 \\
\hline Cuerpos Geométricos & & & & 3.500 & 1.500 & & & 2.000 & $\begin{array}{c}2.000 \\
6.041^{* * *}\end{array}$ & 15.041 \\
\hline Decímetros Cúbicos & & & & & $6.000^{*}$ & $2.500^{*}$ & & 2.000 & $\begin{array}{c}2.000 \\
6.041^{* \star *}\end{array}$ & 16.541 \\
\hline Metros Cúbicos & & & & & 1.500 & 3.500 & & 2.500 & $\begin{array}{l}2.000 \\
6.041^{* * *}\end{array}$ & 16.541 \\
\hline $\begin{array}{l}\text { Bloques Cúbicos } \\
\text { Ladrillo }\end{array}$ & & & & $\begin{array}{r}2.000 \\
80.000\end{array}$ & & & & & & $\begin{array}{r}2.000 \\
80.000\end{array}$ \\
\hline
\end{tabular}

\section{CUADRO A-4}

MATERIALES PRODUCIDOS POR LA MISION PEDAGOGICA ALEMANA

\begin{tabular}{|c|c|c|c|c|c|c|c|c|c|c|}
\hline Materiales & 1970 & 1971 & 1972 & 1973 & 1974 & 1975 & $\begin{array}{l}1975 \\
\text { Dic. }\end{array}$ & 1976 & 1978 & Totales \\
\hline Juegos de mapas & & & 2.000 & 5.000 & & & & 3.000 & $\begin{array}{l}2.000 \\
6.041^{* * *}\end{array}$ & 18.041 \\
\hline Mapas de Colombia & & & & 6.000 & & & & & & 6.000 \\
\hline Sellos de geografía, Juegos & & & $2.000^{*}$ & 1.000 & 2.000 & & & 2.000 & $\begin{array}{l}2.000 \\
6.041^{* \star *}\end{array}$ & 15.041 \\
\hline Mapamundi (globos) & & & 3.000 & 2.000 & 2.000 & & & 2.000 & $\begin{array}{c}2.000 \\
6.041^{\star * *}\end{array}$ & 17.041 \\
\hline Frisos de Historia & & & & 1.000 & & 2.000 & 2.520 & 2.000 & $\begin{array}{c}2.000 \\
6.041^{\star \star *}\end{array}$ & 13.561 \\
\hline Láminas de biología & & & & & & 3.500 & 2.000 & 2.000 & $\begin{array}{c}2.000 \\
6.041^{* * *}\end{array}$ & 15.541 \\
\hline Juegos de seguetas & & & & 8.000 & 1.000 & 1.000 & & 2.000 & $\begin{array}{l}2.000 \\
6.041^{* * *}\end{array}$ & 20.041 \\
\hline Viva la música (disco) & & & 5.000 & & 2.000 & & 5.000 & & & 12.000 \\
\hline $\begin{array}{l}\text { Viva la música } 2 \\
\text { Multiaimnasio de metal }\end{array}$ & & & & & & & & 6.000 & & 6.000 \\
\hline $\begin{array}{l}\text { Multigimnasio de metal } \\
\text { Multigimnasio de madera }\end{array}$ & & & & 80 & & & & & & 80 \\
\hline Multigimnasio de madera & & & & 80 & & & & & & 80 \\
\hline
\end{tabular}

CUADRO A-5

MATERIALES PRODUCIDOS POR LA MISION PEDAGOGICA ALEMANA

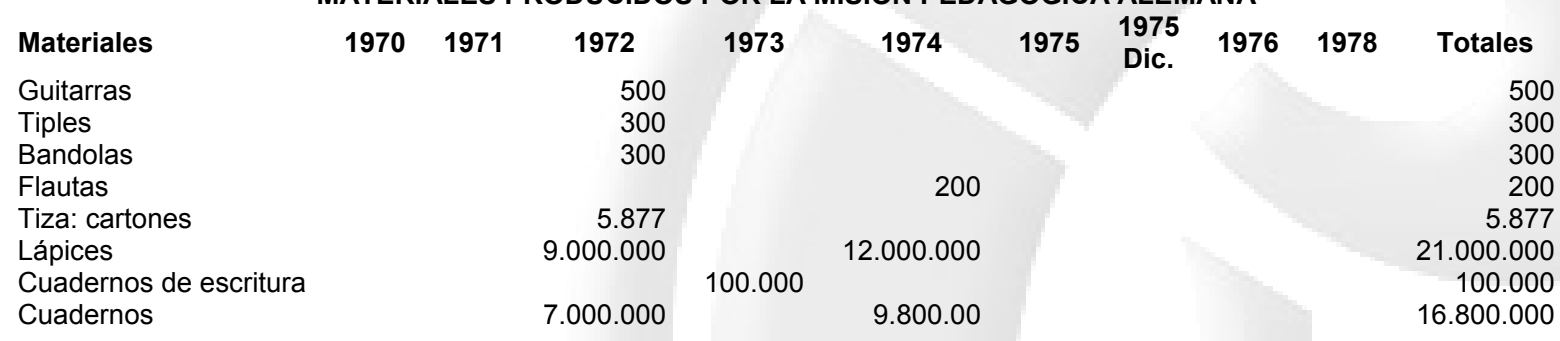


CUADRO 8

\begin{tabular}{|lc|}
\hline \multicolumn{2}{|c|}{ CosTO UNITARIO DE PRODUCCION DE MATERIALES. } \\
\hline \\
\hline
\end{tabular}


CUADRO A-6

\begin{tabular}{|c|c|c|c|c|c|c|}
\hline \multicolumn{7}{|c|}{$\begin{array}{l}\text { EXISTENCIA DE MATERIALES MISION } \\
\text { PEDAGOGICA ALEMANA }\end{array}$} \\
\hline \multicolumn{7}{|c|}{$\begin{array}{c}\text { PORCENTAJES DE ESCUELAS CON MATERIAL DISPONIBLE SEGUN DEPARTAMENTO } \\
\text { POR ZONAS }\end{array}$} \\
\hline \multicolumn{3}{|c|}{ Antioquia } & \multicolumn{2}{|c|}{ Bolívar } & \multicolumn{2}{|c|}{ Boyacá } \\
\hline Materiales & Urbana & Rural & Urbana & Rural & Urbana & Rural \\
\hline Guía Primero & 51.9 & 55.6 & 23.1 & 71.4 & 64.7 & 76.9 \\
\hline Guía Segundo & 66.7 & 88.9 & 38.5 & 71.4 & 41.2 & 53.8 \\
\hline Guía Tercero & 74.1 & 55.6 & 15.4 & 71.4 & 47.1 & 38.5 \\
\hline Guía Cuarto & 59.3 & 88.9 & 34.6 & 85.7 & 64.7 & 38.5 \\
\hline Guía Quinto & 59.3 & 88.9 & 26.9 & 100.0 & 35.3 & 15.4 \\
\hline Calculemos 1 & 51.9 & 77.8 & 30.8 & 28.6 & 29.4 & 7.7 \\
\hline Guía Calculemos 1 & 44.4 & 66.7 & 15.4 & 14.3 & 5.9 & 0.0 \\
\hline Calculemos 2 & 48.1 & 88.9 & 50.0 & 28.6 & 29.4 & 15.4 \\
\hline Guía Calculemos 2 & 59.3 & 77.8 & 19.2 & 14.3 & 5.9 & 7.7 \\
\hline Calculemos 3 & 48.1 & 100.0 & 42.3 & 14.3 & 29.4 & 30.8 \\
\hline Guía Calculemos 3 & 51.9 & 66.7 & 46.2 & 0.0 & 17.6 & 15.4 \\
\hline Calculemos 4 & 25.9 & 44.4 & 30.8 & 0.0 & 17.6 & 7.7 \\
\hline Guía Calculemos 4 & 37.0 & 11.1 & 30.8 & 14.3 & 1.7 & 7.7 \\
\hline Calculemos 5 & 25.9 & 33.3 & 3.8 & 28.6 & 23.5 & 15.4 \\
\hline Guía Calculemos 5 & 29.6 & 33.3 & 7.7 & 14.3 & 17.6 & 15.4 \\
\hline Cancionero 1 & 33.3 & 77.8 & 34.6 & 42.9 & 17.6 & 7.7 \\
\hline Cancionero 2 & 29.6 & 33.3 & 23.1 & 28.6 & & \\
\hline Clase Alegre 1 & 22.2 & 88.9 & 0.0 & 28.6 & 17.6 & 7.7 \\
\hline Clase Alegre 2 & 25.9 & 66.7 & 7.7 & 14.3 & & \\
\hline Edufísica 1 & 55.6 & 55.6 & 42.3 & 14.3 & 29.4 & 0.0 \\
\hline Edufísica 2 & 63.0 & 66.7 & 42.3 & 42.9 & 29.4 & 23.1 \\
\hline Juegos Atléticos & 7.4 & 0.0 & & & & \\
\hline Vamos a Leer & 25.9 & 22.2 & 23.1 & 0.0 & 29.4 & 30.8 \\
\hline Libro de Geografía & 3.7 & 33.3 & 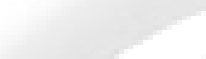 & & & 7.7 \\
\hline Sellomat & 55.6 & 78.8 & 46.2 & 46.9 & 47.1 & 100.0 \\
\hline Fracciograf & 48.1 & 88.9 & 61.5 & 42.9 & 11.8 & 30.8 \\
\hline$M^{3}$ & 48.1 & 66.7 & 30.8 & 42.9 & 52.9 & 100.0 \\
\hline $\mathrm{Dm}^{3}$ & 40.7 & 66.7 & 11.5 & 28.6 & 41.2 & 76.9 \\
\hline Figuras Geométricas & 51.9 & 77.8 & 34.6 & 42.9 & 58.8 & 76.9 \\
\hline Ficheros & 55.6 & 88.9 & 50.0 & 0.0 & 58.8 & 100.0 \\
\hline Lotelect & 37.0 & 100.0 & 11.5 & 42.9 & 58.8 & 84.6 \\
\hline Sellolect & 37.0 & 77.8 & 11.5 & 28.6 & 52.9 & 61.5 \\
\hline Mapa Político & 29.6 & 88.9 & 15.4 & 57.1 & 58.8 & 61.5 \\
\hline Mapa Físico & 25.9 & 88.9 & 19.2 & 28.6 & 47.1 & 61.5 \\
\hline Mapa Físico Suramer. & 33.3 & 77.8 & 7.7 & 42.9 & 58.8 & 61.5 \\
\hline Mapa Político Suramer. & 25.9 & 88.9 & 26.9 & 42.9 & 58.8 & 61.5 \\
\hline Mapa Físico Colombia & 25.9 & 66.7 & 23.1 & 42.9 & 58.8 & 53.8 \\
\hline Mapa Político Colombia & 29.6 & 55.6 & 26.9 & 57.1 & 64.7 & 61.5 \\
\hline Globo & 29.6 & 77.8 & 7.7 & 14.3 & 58.8 & 76.9 \\
\hline Sellos Geografía & 44.4 & 66.7 & 53.8 & 42.9 & 52.9 & 92.3 \\
\hline Frisos Historia & 29.6 & 66.7 & 38.5 & 42.9 & 41.2 & 61.5 \\
\hline Láminas Biología & 29.6 & 77.8 & 57.7 & 71.4 & 58.8 & 61.5 \\
\hline
\end{tabular}




\section{BIBLIOGRAFIA}

CAIN, B. B. and ROBINSON HOLLISTER, "The methodology of Evaluating Social Action Programs", in Peter H. Rossi (Ed.), Evaluating Social Programs, New York, Seminar Press, 1972.

CARO. FRANCIS G. (Ed.> Readings in Evaluator Research, New York: Russell Sage Foundation, 1971.

CEBALLOS NIETO, DANIEL, "Algunas consideraciones previas sobre la cooperación técnica internacional en el Sector Educativo, en: Documentación Educativa, UPN, Volumen 4, No. 16.

CONVENIO COLOMBO ALEMAN, 1965.

CONVENIO ADICIONAL COLOMBO ALEMAN, 1968.

DANE, Boletín Mensual de Estadísticas, Nos. 288 y 311.

DECRETO 1710 de julio 25 de 1963.

DECRETO 150 de 1967.

DECRETO 1419 de 1976.

DECRETO 088 de 1976.

DEPARTAMENTO NACIONAL DE PLANEACION, Estudio de la Eficiencia Interna de la Educación Primaria, 1961.

DEPARTAMENTO NACIONAL DE PLANEACION, Planes y Programas 1969-1972". Bogotá, Dic. 1969.

FONTAINE, ERNESTO, Evaluación Social de Proyectos. Publicación de la Escuela Superior de Administración Pública.

FREEMAN, II. "The present status of Evaluation Studies", in M. Guttentag and 5. Suar (Ed), Evaluation Studies, Riview annual, Vol. 2, Beverly Hills: Sage Publications, 1977.

GREENBERG, B.G., Evaluations of Social Programs”, en Francia G. Caro. (op. cit.).

HEINO, ULMER, Abschlussberich Über dic Nachbetreuungsphase 1976 -1978, Bogotá, Oktober, 1978.

ICFES - MEN, La Educación en Cifras 1970 - 1974, Bogotá, Diciembre de 1975.

INSTITUTO ARNOLD-BERGSTRAESSER, La Misión Pedagógica Alemana y la Reforma en la Escuela Primaria Colombiana. 
JAMISON, DEAN T. et al, Cost Analysis for Educational Planning and Evaluation: Methodology and Aplication to Instructional Technology, A.I.D., 1976.

JARAMILLO DUQUE, JAIME. El proceso de la Educación del Virreynato a la Época Contemporánea, Tomo III, 1980.

KAESSMANN, WALTER, Abschlussbericht Über das Projekt FE 898 (652527/3) Innere Reform des Kolumbianischen Primarschulwesens. Bogotá, septiembre, 1975.

KAESSMANN, Walter, "Diagnóstico sobre la situación actual de la Enseñanza de Matemáticas en la Escuela Primaria de Colombia".

MINISTERIO DE EDUCACION NACIONAL, La Educación Primaria en Colombia 19601969. Mimeógrafo. Bogotá. 1972.

MINISTERIO DE EDUCACION NACIONAL, Anexo Estadístico 1960-1968. Mimeógrafo. Bogotá, 1970.

MINISTERIO DE EDUCACION NACIONAL, Capacitación del Magisterio, División de Capacitación del Magisterio. Bogotá. 1970.

MINISTERIO DE EDUCACION NACIONAL, Política Educativa, Tomos I y II.

MOJICA. GUILLERMO, Problemas y Perspectivas de la Educación en Colombia, Mimeógrafo, Circulación Restringida, 1974.

RAMIREZ VARELA, DELIO, La Misión Pedagógica Alemana ante el Ministerio de Educación de Colombia1965-1978”,. Bogotá. Enero, 1978.

RODRIGUEZ ROJAS, JOSE MARIA. Panorama de la Educación Colombiana. Desarrollo del Programa Oficial para el 6o. año de Escuelas Normales, Bedout, 1a. Edic., 1963.

SALAZAR, MARIA CRISTINA, "Elementos Pedagógicos para la Educación Primaria en las áreas rurales", en Revista Colombiana de Educación, UPN, II Semestre, 1978.

STUFLEBEAM, D., Educational Evaluation decision-making, P.D.R. National Studies Committee on Evaluation.

SUCHMAN, EDWARD, Evaluative Research, Russell Sage Foundation, New York, 1967.

TORRES, FERNAN Y PEREIRA FRANCISCO. Modelos de Flujos, Informe Final. Doc. ICOLPE 149/IE. 98/IX/73. Mimeógrafo. 